\title{
Selbstgerechtigkeit \\ Selbstermächtigte Status-Degradierungszeremonien von \\ Online-Petitionen bis zum Lynchen 2.0
}

\author{
Wolf-Andreas Liebert (Koblenz)
}

\begin{abstract}
An increasing lack of commitment and brutalization was observed due to the erosion of traditional communities in late modernism (cf. Duerr 1993; Sennet 1998). In Social Media, this is shown as an aggressive style (flaming) or as "hate speech" (cf. Butler 2013), increasingly in organized forms of ensembles of actors of aggression directed specifically against individuals. This libel and these hate acts underlie a complex practice which can be described with Harold Garfinkel's concept as "status degradation ceremony". Garfinkel's terms refer to court cases, i. e. an institutionalized form of aggression exercising - according to Sigmund Freud's terms one of the main benefits of every culture in order to prevent violent squalor and brutality. At the same time, however, Freud also expressed unease ("Unbehagen") that this aggression could more or less break with institutional failure. Today, such processes can be increasingly observed in a bandwidth ranging from simple online petitions to radical forms of vigilantism.

In order to understand why individuals take the law into their own hands and - often associated with a more or less large swarm formation - why they are trying to define law, to judge, and even to execute their own "judgments" outside of the democratically constituted legal system, a second concept is introduced, the concept of Selbstermächtigung which refers to practices of autonomy. This concept of Selbstermächtigung is understood as a time diagnostic term to describe a number of communicative practices which could jeopardize democracy in one of its institutional foundations in question: its legal constitution. Selbstermächtigung describes a contemporary mentality which contains a strong, emotionally charged tension between the individual and the institution namely in all sorts of social institutions, such as in health, science, but also democracy - which only just enables these criticism of institutions. From the two analytical concepts of Selbstermächtigung and the status degradation ceremony, we can develop the concept of a "self-authorized status degradation ceremony". With this concept, we can examine both simple online petitions and extreme forms of vigilante justice, which are similar to lynching. This is done plausible on the basis of exemplary analyzes. Finally, the danger is clear that the anti institutionally polarized mentality of Selbstermächtigung exactly threatens the institution that enables them.
\end{abstract}




\section{$1 \quad$ Einleitung}

Mit der Erosion tradierter Gemeinschaften in der Spätmoderne wurde eine zunehmende Bindungslosigkeit und Verrohung beobachtet (vgl. Duerr 1993; Sennet 1998); in den Neuen Medien zeigt sich dies als aggressiver Stil (Flaming) oder in der so genannten hate speech (vgl. Butler 2013). Zu diesen eher unorganisierten Praktiken gesellen sich aber zunehmend organisiertere Formen, in denen Akteur-Ensembles Aggressionen gezielt gegen Einzelpersonen richten. Diese Beleidigungs- und Hass-Akte lassen eine komplexe Praktik erkennen, die sich mit einem Begriff von Harold Garfinkel (1956) als so genannte StatusDegradierungszeremonie beschreiben lässt. Garfinkels Ausführungen beziehen sich auf Gerichtsverhandlungen, d. h. es handelt sich um eine institutionell abgesicherte Form der Aggressionsausübung, was in der Kulturtheorie Sigmund Freuds (1930/2004) eine der Hauptleistungen jeder Kultur zur Verhinderung gewalttätiger Verwahrlosung und Verrohung darstellt. Zugleich äußerte Freud jedoch auch ein Unbehagen, dass sich bei einem Versagen der Institution diese Aggressionen mehr oder weniger unkontrolliert Bahn brechen könnten. Heute lassen sich solche Prozesse - in einer Bandbreite, die von einfachen Petitionen bis zu radikalen Formen der Parallel- oder Selbstjustiz reichen - zunehmend beobachten.

Um zu verstehen, warum zunehmend Einzelpersonen das Recht selbst in die Hand nehmen und - häufig mit einer mehr oder weniger großen Schwarmbildung verbunden - versuchen, außerhalb des demokratische verfassten Rechtssystems Recht zu definieren, zu sprechen und auch zu exekutieren, wird ein zweiter Begriff eingeführt, der Begriff der Selbstermächtigung. Dieser Begriff der Selbstermächtigung soll als zeitdiagnostischer Begriff verstanden werden, um eine Reihe von Entwicklungen kommunikativer Praktiken zu beschreiben, die die Demokratie in einer ihrer institutionellen Grundfesten in Frage stellen könnte, nämlich in ihrer rechtsstaatlichen Verfasstheit. Wenn ein Begriff wie Selbstermächtigung von vielen Diskursteilnehmern aktiv und recht unterschiedlich verwendet wird, so könnte gefragt werden, ob dieser Begriff als analytischer Begriff überhaupt zulässig sein könne, wenn er doch offensichtlich diskursrelvant sei. Bei genauerem Hinsehen sind jedoch eine Vielzahl von analytischen Begriffen sowohl Teilnehmerkategorien als auch wissenschaftlich-analytische Begriffe, nicht zuletzt der Begriff der Demokratie selbst. Es kommt vielmehr darauf an, die wissenschaftlich zu verwendenden Begriffe wie Selbstermächtigung in ihrer Bedeutung zu fixieren, damit sie im wissenschaftlichen Kontext (mehr oder weniger) eindeutig verstanden werden können. Eine Definition ist daher von Nöten, ist sie jedoch erbracht, kann man sich zwar über diese Definition streiten, das Problem, dass derselbe sprachliche Ausdruck auch im Diskurs in vielfältiger Weise verwendet wird, ist dann jedoch obsolet. Die Definition von Selbstermächtigung, die später gegeben werden wird, wird eine Mentalität in der Gesellschaft beschreiben, in der sich eine starke, teilweise emotional geladene Spannung zwischen Individuum und Institution aufgebaut hat und zwar in allen möglichen gesellschaftlichen Institutionen, wie im Bereich Gesundheit, der Wissenschaft, aber auch der Demokratie, die diese InstitutionenKritik ja in grundlegender Weise erst ermöglicht.

Aus den beiden analytischen Begriffen der Selbstermächtigung und der StatusDegradierungszeremonie lässt sich das Konzept der selbstermächtigten StatusDegradierungszeremonie entwickeln, das dann geeignet ist, sowohl einfache OnlinePetitionen als auch extreme Formen lynchähnlicher Selbstjustiz zu untersuchen. Die im Fol- 
genden besprochenen Beispiele weisen auf die Gefahr hin, dass die antiinstitutionell polarisierte Mentalität der Selbstermächtigung genau die Institution gefährdet, die sie ermöglicht. Ein Blick auf Ungarn und vergleichbare Konstellationen zeigt, dass diese Gefahr durchaus real ist.

Die hier vorgestellten Fallstudien können nur exemplarischen Charakter besitzen, es geht hier darum, das Prinzipielle aufzuzeigen, das "Unbehagen" deutlich werden zu lassen, das am Ende der Arbeit mit Bezug zu Sigmund Freud herausgestellt werden soll.

Zunächst sollen jedoch die linguistischen Ausgangspunkte benannt werden, an denen eine Untersuchung von selbstermächtigten Status-Degradierungszeremonien ansetzen kann. ${ }^{1}$

\section{Sprache, Demokratie und Aggression als Themen linguistischer Forschung}

Linguistische Fragestellungen in Bezug auf das Thema Demokratie bzw. das Thema Demokratie und Sprache wurden insbesondere in den bekannten Studien der kritischen Diskursanalyse und der Düsseldorfer Schule untersucht. In der kritischen Diskuranalyse, z. B. bei Ruth Wodak, Siegfried Jäger oder Jürgen Link, wurde insbesondere die Rezeption Michel Foucaults zu einem wichtigen Bezugspunkt. Dem gegenüber entwickelte die Düsseldorfer Schule, also die Arbeiten in der Tradition von Georg Stötzel, später Dietrich Busse, Wolfgang Teubert, Martin Wengeler, Thomas Niehr und anderen, einen eigenen Diskursbegriff, der auf textübergreifende gesellschaftliche, thematisch gebundene Diskussionszusammenhänge abzielte. In den Anfängen wurden hier anhand von Sprachthematisierungen neuartige Wörterbücher nach dem Schlüsselwortprinzip entwickelt, am bekanntesten sicherlich die "Kontroversen Begriffe" (Stötzel/Wengeler 1995). Später wurden auch größere Diskurszusammenhänge analysiert (vgl. z. B. Wengeler/Ziem 2013). Dabei stand die historisch-semantische Analyse von Argumentationszusammenhängen, vor allem in Form von Topoi, im Vordergrund; in jüngster Zeit auch in Verbindung mit korpuslinguistischen Methoden. Einen verbindenden Weg schlug Heidrun Kämper ein, die versuchte, eine zeitgeschichtliche, demokratiespezifische Semantik auszumachen, die sie etwa in ihrem Werk "Wörterbuch zum Demokratiediskurs 1967/68" aus dem Jahr 2013 beschrieb, und dabei sowohl auf die kritische Diskursanalyse als auch auf die Düsseldorfer Schule zurückgriff.

Neben diesen explizit die Demokratie thematisierenden Arbeiten, gab es weitere, verwandte Arbeiten, zum einen aus der sprachkritisch motivierten Rechtslinguistik (vgl. Müller/Wimmer 2001; Felder 2003; Vogel 2012), zum anderen aus der Gesprächsforschung, beispielsweise die Beschreibung aggressiven Kommunikationsverhaltens (vgl. Kallmeyer/Schmitt 1996). Für die neueren Formen des informell organisierten, aggressiven Kommunizierens ist aber keine der genannten Arbeiten ausgewiesen, obwohl sich sicher Ansatzpunkte finden lassen.

Im Folgenden soll ein Konzept von Harold Garfinkel, die Status-Degradierungszeremonie eingeführt werden, das in der Linguistik noch relativ wenig bekannt ist, obwohl Garfinkels Ansatz der Ethnomethodologie in der Linguistik als sehr etabliert gelten kann. ${ }^{2}$

1 Für Fragen, kritische Diskussionen u. a., die zu diesem Artikel beigetragen haben, bedanke ich mich bei folgenden Personen: Stephan Habscheid, Thomas Niehr, Martin Reisigl, Pamela Steen, Helmuth Feilke, Ruth Wodak, Clemens Knobloch, Friedemann Vogel, Tanja Gnosa, Theresa Haas, Nicolai Glasenapp, Jihee Hong, Sarah Weber, Miriam Spurzem, Winfried Gebhardt, Kathrin Fischer, Nicole Mattern. 
Später wird sich dann zeigen, dass mit dem Konzept der Status-Degradierungszeremonie viele Fälle erfasst werden, aber nicht in ihrer Kulturbedeutung erfasst werden können. Dafür soll dann später das Makro-Konzept der Selbstermächtigung eingeführt werden, mit dem die Relevanz von Status-Degradierungszeremonien besser eingeschätzt werden kann.

\section{Der Begriff der Status-Degradierungszeremonie}

Harold Garfinkel hat seinen Begriff der Ethnomethodologie, der schließlich paradigmatisch für viele Disziplinen werden sollte, u. a. aus seinen Forschungsbeobachtungen von Gerichtsverfahren, insbesondere auch bei der Meinungsbildung von Geschworenen, entwickelt. In diesem Zusammenhang entstand auch der Begriff der Status-Degradierungszeremonie. Nach Garfinkel (1956: 420) muss jede Kultur, die nicht in "Barbarei ausartet", über institutionell abgesicherte Verfahren verfügen, die regeln, wie normverletzendes Verhalten erkannt, bewertet und bestraft wird. Was eine Status-Degradierungszeremonie sei, definiert Garfinkel allgemein wie folgt:

Any communicative work between persons, whereby the public identity of an actor is transformed into something looked on as lower in the local scheme of social types, will be called a 'status degradation ceremony'.

(Garfinkel 1956: 420)

Es handelt sich also um einen sprachlich-kommunikativen Prozess, der zumindest eine gewisse Öffentlichkeit voraussetzt. Es gibt einen Ankläger und einen Angeklagten und nach einer im performativen Sinne - erfolgreichen Zeremonie ist der Angeklagte einem herabsetzenden Statuswechsel unterzogen, der zugleich einen Identitätswechsel bedeutet, also etwa: ein gerade noch respektabler Bürger ist im nächsten Moment ein verachtungswürdiger Verbrecher. Es ist daher kein Wunder, wenn die Gerichtsverhandlung mit einer massiven Verwandlung der sozialen Identität als prototypisch für Status-Degradierungszeremonien gilt. Garfinkel selbst hat aber bereits darauf hingewiesen, dass sich solche Status-Degradierungen in allen Gesellschaften, in denen ein normatives Konstrukt wie Moral existiert, finden lassen. Worauf es also ankommt, ist die Ausbildung eines institutionell geregelten Verfahrens für StatusDegradierungszeremonien, die dann im Bedarfsfall aktiviert werden können. Die Gerichtsverhandlung mit allen damit zusammenhängenden kulturellen Konstrukten ist eben nur die Form, wie sie sich in unseren Gesellschaften herausgebildet hat. Prototypisch setzt Garfinkel für eine Status-Degradierungszeremonie bestimmte Voraussetzungen bzw. Prozessschritte an: Notwendig ist ein Ankläger, der öffentlich beansprucht, als Repräsentant für eine Gruppe zu sprechen, und der glaubwürdig überpersönliche Werte vertritt und verteidigt, also eine Moral, Ethik oder der im Gerichtsverfahren für ein Gesetz sprechen kann. Weiterhin benötigt man die Rolle des Angeklagten/Sünders, eines wie auch immer moralisch Verwerflichen, dem anhand eines konkreten Ereignisses oder Falles nachgewiesen werden soll, dass unter seiner Maske etwas anderes verborgen ist, was in fundamentaler Weise gegen diese als verbindlich geltenden Werte verstößt. Dabei ist es weniger entscheidend, was er im Einzelnen getan hat, sondern was seine "wahren" Gründe und Motive für die Tat bzw. Taten waren. Die Verwerf-

\footnotetext{
2 Einzelne Arbeiten sind natürlich vorhanden. So hat etwa Arnulf Deppermann (2005: 268) in Anlehnung an Garfinkel einen Degradierungsbegriff entwickelt, der keinen massiven Identitätswechsel voraussetzt, sondern auch abgeschwächte Formen erlaubt, etwa eine Faceverletzung.
} 
lichkeit der Motive und Antriebe steht metonymisch für die Verwerflichkeit der gesamten Person. Ziel ist es, ein Persönlichkeitsmodell zu schaffen, bei der aus diesen verwerflichen Motiven dann die Tat plausibel erklärt werden kann. Dieses Persönlichkeitsmodell ist dann zugleich die neue subalterne Identität, die dem Verurteilten dann "im Erfolgsfall" zugewiesen werden wird. Wenn also die "wahre" Motivik nachgewiesen werden kann, ist performativ seine wahre neue Identität bereits hergestellt und damit auch zugleich die Degradierung vollzogen, so dass der Verurteilte ein "anderer Mensch" ist. Die Degradierung setzt eine moralische Entrüstung und Empörung über die Tat voraus, die in eine öffentliche Anklage mündet. Dabei muss der Angeklagte als ein außergewöhnlicher Fall, als besonders abweichend vom Alltäglichen dargestellt werden.

Garfinkel beschreibt dies als folgendes Muster:

The paradigm of moral indignation is public denunciation. We publicly deliver the curse: "I call upon all men to bear witness that he is not as he appears but is otherwise and in essence" of a lower species.

(ebd.: 421)

Das Prinzip moralischer Entrüstung ist nach Garfinkel also das öffentliche Anprangern und Anklagen. Öffentlich wird eine Art Fluch ausgesprochen: "Ich fordere alle auf, zu bezeugen, dass diese Person nicht so ist, wie es den Anschein hat, sondern dass sie ganz anders ist und zwar von niederer Art." Ziel der Degradierung ist die rituelle Zerstörung der Identität der Person. Die moralische Entrüstung stärkt die Gruppenbindung der Normvertreterseite und schafft ein starkes Band der Gemeinsamkeit. Damit eine Degradierungszeremonie erfolgreich sein kann, muss der Anprangernde oder Ankläger außerdem zeigen, dass er nicht aus persönlichen Beweggründen vorgeht, sondern im Namen einer übergeordneten Sache, Moral usw. spricht. Der Ankläger muss eine Nähe zu den Zeugen suchen und einen möglichst großen Abstand zum Angeklagten herstellen, diesen möglichst fremdartig darstellen, als außerhalb der normalen Ordnung stehend. Die performativ erfolgreiche Status-Degradierung hängt nach Garfinkel weiterhin von einer Neudefinition des Täters und des Ereignisses ab. Und zwar müssen sowohl der Täter als auch seine Tat oder das Ereignis vom Alltäglichen scharf abgegrenzt und in das Außergewöhnliche gerückt werden. Ein weiteres Element, das Garfinkel anführt, wird gleich noch einen wichtigen Unterschied ausmachen: Bei Garfinkel muss der Ankläger, wie bereits beschrieben, in der Öffentlichkeit oder in der Gruppe als offensichtlicher Vertreter, ja sogar Verfechter dieser Werte/Moral angesehen werden. Dies stellt sich in den sozialen Medien anders dar und soll in den Fallanalysen deutlich gemacht werden.

Im nächsten Schritt soll nun der Begriff der Selbstermächtigung eingeführt werden, um daraus dann das Konzept der selbstermächtigten Status-Degradierungszeremonie zu gewinnen.

\section{Selbstermächtigung als zeitdiagnostischer Begriff}

Ein wesentliches Charakteristikum spätmoderner Gesellschaften besteht in einem grundsätzlich veränderten Verhältnis von Individuum und Institution, das durch große Spannungen geprägt ist. Um dieses Spannungsverhältnis zu erfassen, soll der Begriff Selbstermächtigung eingeführt werden.

In Anlehnung an Albrecht et al. (2012) soll unter dem Begriff der Selbstermächtigung ein Deutungsmuster verstanden werden 
für eine sich ausbreitende normativ-politische (Protest-)Einstellung in "spätmodernen" Gesellschaften (...). Er umreißt eine generelle Unzufriedenheit mit den institutionellen Rahmenbedingungen, den damit verbundenen Machthierarchien und der daraus resultierenden "Entmündigung" des "normalen Bürgers" und fordert - selbstbewusst und offensiv - dazu auf, sein Schicksal in die eigene Hand zu nehmen, seine individuellen Ansprüche nicht nur zu formulieren, sondern auch - aktiv - durch "Selbsttätigkeit" umzusetzen, - auch wenn dies bedeutet, gegen die eingespielten Regeln und etablierten Strukturen der institutionellen Ordnungen zu verstoßen. Noch mehr: Selbstermächtigung heißt zunehmend, die vorgegebenen Institutionen und damit die institutionelle Ordnung "spätmoderner" Gesellschaften bewusst in Frage zu stellen (...).

(Albrecht/Gebhardt/Geulen/Liebert 2012)

Als "neues Modell" findet sich kein ausgearbeitetes neues Gesellschaftsmodell, es geht hier also nicht um neue "Große Erzählungen" (vgl. Lyotard 1999), die zur Überwindung der Institutionen etabliert werden, sondern eher um vage und diffuse Vorstellungen von einer besseren Sozialität.

Diese Definition steht dabei nicht nur in einem geistesgeschichtlichen (vgl. Liebert 2015), sondern auch einem geisteswissenschaftlichen Kontext: Vielfach bereits in literaturwissenschaftlichen Interpretationen im Sinne von Hybris verwendet, wurde der Begriff der Selbstermächtigung als wissenschaftlich-analytischer Begriff zuerst in der Religionssoziologie etabliert (vgl. Bochinger/Engelbrecht/Gebhardt 2009). Dort soll er das Phänomen erfassen, dass die zu beobachtende Häufung von Kirchenaustritten - und damit die Abwendung von der Institution - in der Regel nicht auch eine Abkehr vom Glauben bedeuten. Vielmehr nimmt das "religiöse Subjekt" sich nun das Recht heraus, seinen Glauben zu individualisieren, mit unterschiedlichsten Metaphysiken zu kombinieren und nimmt sich auch selbst das Recht, also an den dafür vorgesehenen ermächtigten Amtsträgern vorbei, "Amtshandlungen" vorzunehmen, etwa die Eucharistie zu feiern. Ein aktuelles Beispiel für Letzteres ist der Fall von Martha Heizer, der Vorsitzenden von "Wir sind Kirche." in Österreich. Da sie die Eucharistie ohne Pfarrer selbst durchführte, wurde sie exkommuniziert. Eine Innsbrucker Pfarrerinitiative protestierte zwar gegen die Exkommunikation, bezog aber zugleich Position "gegen die Selbstermächtigung von christlichen Gemeinschaften und Einzelpersonen zur Feier der Eucharistie" (DiePresse.com 2014). Die Angst der Institution, dass durch eine zu weitreichende Toleranz von Selbstermächtigungen sich ihre Identität ins Verschwommene auflöst, ist hier deutlich spürbar.

Aber nicht nur im Bereich der Religion, sondern in praktisch allen gesellschaftlichen Bereichen lassen sich Formen der Selbstermächtigung zeigen, sei es im Bereich Wissenschaft, der Gesundheit und Medizin, im Bereich Tod und Sterben und natürlich auch im politischen Bereich. Der "Aufstand der Laien" (Kerner 1996) kann dabei auch zur Bildung von Parallelinstitutionen führen, die in Konkurrenz zu den etablierten Institutionen, auch staatlichen, treten. Bekannt sind etwa die vergemeinschaftenden Formen einer Allmende-Politik für eine neue Kultur des Teilens, die mittlerweile einen eigenen rechtlichen Rahmen in Form eigener Nutzungslizenzen, den so genannten "Creative Commons", geschaffen haben und als Alternative zur Politik des geistigen Privateigentums antreten (vgl. Grassmuck 2002) oder die neuen experimentelleren Formen einer "liquid democracy".

Es wäre allerdings - auch mit Blick auf die oben ausgeführte Definition - ein Missverständnis, Selbstermächtigung mit Emanzipation gleichzusetzen, denn das grundlegende Span- 
nungsverhältnis von Individuum und Staat liegt quer zu herkömmlichen politischen Einteilungen und kann daher auch zu neuen - durchaus flüchtigen - Konstellationen und Gruppierungen kommen wie beispielsweise die "Patriotische Europäer gegen die Islamisierung des Abendlandes" (Pegida). Es handelt sich daher eher um eine Mentalität (vgl. Gebhardt 1993; Hermanns 1995), die sich durchgängig in der Gesellschaft finden lässt. Die oben angeführte Definition ist also insofern zu ergänzen, dass der utopische Gehalt, der mit der empörtprotesthaften Haltung einhergeht, offen zu fassen ist, sodass nicht nur emanzipative Formen der Selbstorganisation, sondern auch Phantasien einer Rückkehr zu einer ursprünglich "reinen", aber vom Verlust bedrohten oder bereits verlorenen Identität.

Auf der Vergemeinschaftungsebene hat die Mentalität der Selbstermächtigung zur Folge, dass sich die Einzelnen in informellen Organisationsformen wie Netzwerken zusammenfinden mit relativ unverbindlicher, variabler Partizipation. Scheinbar unvorhersehbar treten sie in Aktion, feste Führungsstrukturen sind selten vorhanden, eher verschiedene Aktivisten, die sich aber auch durchaus für die jeweilige Aktion als Machtzentren oder führende Organisatoren etablieren können ${ }^{3}$. Das Angebot an neuen Medien und die Nachfrage in Kulturen der Selbstermächtigung verstärkt sich dabei wechselseitig, so dass kommunikative Praktiken der Selbstermächtigung zu einem wesentlichen Teil in den Neuen Medien stattfinden.

\section{$5 \quad$ Selbstermächtigte Status-Degradierungszeremonien}

Garfinkels Konzept der Status-Degradierungszeremonie stellt zunächst eine notwendige Einrichtung jeder Kultur dar, um in institutionell geregelter Weise und im Namen eines institutionell konstituierten Rechts öffentlich Einzelne aus der Gemeinschaft herauszuheben, ihren niederen, aber eigentlichen Charakter aufzudecken, und die in ihrem Status als gesellschaftliches Mitglied - im Sinne einer Statusherabstufung - zu platzieren.

Unterstellt man nun die spätmoderne Mentalität der Selbstermächtigung, also das starke Misstrauen gegen die demokratische Institution des Rechts, Empörung über gefällte (oder eben nicht gefällte) Urteile, Misstrauen gegen die Unabhängigkeit der Gerichte, Unzufriedenheit mit der Gesetzgebung, einhergehend mit dem Glauben, es besser zu können, dann liegt es dieser Mentalität nahe, die demokratisch-juristischen Institutionen nicht nur zu kritisieren, sondern vielmehr - im Glauben an die Eigenkompetenz, Recht besser als die zuständige Institution verstehen und auch sprechen zu können - dieses Recht und die damit einhergehende Status-Degradierung auch unter Missachtung und außerhalb der juristisch relevanten Institutionen eigenhändig herzustellen.

Damit ist aber ein Kern der Demokratien berührt, die das Prinzip der Rechtsstaatlichkeit zum konstitutiven Kern ihrer Verfassung machen, und daraus ein Gewaltmonopol des demokratisch legitimierten Staates ableiten, was jegliche Form der Selbstjustiz kategorisch ausschließt.

So kann nun der Begriff der selbstermächtigten Status-Degradierungszeremonie folgendermaßen definiert werden:

3 Dazu gehören auch die so genannten Flashmobs (vgl. etwa Gebhardt/Waldeyer 2011). 
Wenn ein Akteur (Person, Personengruppe, Schwarm, Organisation) ein Verhalten eines anderen Akteurs als normverletzend identifiziert und sanktionieren will und nicht die Institutionen und institutionell geregelten Status-Degradierungszeremonien nutzt, sondern eine eigene Status-Degradierungszeremonie außerhalb der vorgesehenen Institutionen nur durch sich selbst ermächtigt durchführt, dann soll dies eine selbstermächtigte StatusDegradierungszeremonie genannt werden.

Dabei können bei einer selbstermächtigten Status-Degradierungszeremonie durchaus Abläufe der entsprechenden institutionell geregelten Form simuliert werden. So tritt ein "Ankläger" (eventuell mit einer Anzahl an Unterstützern) als Repräsentant einer größeren Gruppe (etwa für die "Zuschauer", die "Gebührenzahler", die "Steuerzahler" oder das "Volk"), von der er aber nicht gewählt wurde, oder auch im Namen einer höheren moralischen Instanz oder Idee auf. Man kann diesen Akt als selbstermächtigte Repräsentation bezeichnen, bei der in einem Als-ob-Modus so getan wird, als gäbe es institutionelle Verfahren, durch die der selbstermächtigte Ankläger als Repräsentant legitimiert sei. Letztlich aber bleibt es eine Simulation institutioneller Verfahren, obwohl eine tatsächliche Statusdegradierung der angegriffenen Person realisiert werden kann und in den meisten Fällen auch wird. ${ }^{4}$ Welche StatusDegradierung für den Einzelnen dann tatsächlich eintritt, ist jedoch schwer kalkulierbar, es ist im geringsten Fall eine Rufschädigung, einhergehend mit dem Verlust von symbolischem und dann auch realem Kapital, etwa durch ausbleibende Aufträge, Engagements usw.

Es kann dabei nicht selten die Situation entstehen, dass selbstermächtigte StatusDegradierungszeremonien sogar in Konkurrenz zu den institutionellen Verfahren treten, so dass durchaus der Fall eintreten kann, dass obwohl der reguläre Angeklagte im regulären Gerichtsverfahren freigesprochen wird, also durch die Institution keine Statusdegradierung erfährt, der gerichtliche Freispruch jedoch nichts daran ändert, dass die parallel dazu geführte selbstermächtigte Status-Degradierungszeremonie zu einem anderen Ergebnis kommt und der vor Gericht Freigesprochene eine (selbstermächtigte) Status-Degradierung erfährt.

\section{Fallanalysen}

Im Folgenden sollen nun einige Fallbeispiele selbstermächtigter Status-Degradierungszeremonien besprochen werden, die von einfachen Online-Petitionen bis zu massiven lynchähnlichen Angriffen reichen. Etwas ausführlicher soll zunächst das Beispiel der Reaktionen auf die "Dresdner Rede" der Schriftstellerin Sibylle Lewitscharoff und dann als weitere Beispiele die Online-Petition gegen Markus Lanz, die Online-Aktionen gegen verschiedene ProfessorInnen und schließlich gegen No-names, die einer moralisch verwerflichen Tat verdächtig sind, behandelt werden.

\footnotetext{
4 Dies entspricht dem aus dem Symbolischen Interaktionismus bekannten Thomas-Theorem "If men define situations as real, they are real by its consequences" (Thomas/Thomas 1928: 572).
} 


\subsection{Selbstermächtigte Degradierungszeremonien nach der "Dresdner Rede" von Sibylle Lewitscharoff}

\subsubsection{Kontext und Einordnung der Rede Lewitscharoffs}

Am 02.03.2014 hielt die Schriftstellerin Sibylle Lewitscharoff einen Vortrag in der Reihe "Dresdner Reden" im Schauspielhaus Dresden. In dieser Ansprache nahm sie auf der Oberfläche Stellung zur Reproduktionsmedizin und zwar in einer Weise, die sie ins Zentrum der medialen Aufmerksamkeit katapultierte (vgl. Lewitscharoff 2014).

Es ist durchaus nicht einfach, die Rede Lewitscharoffs als Genre zu charakterisieren. So könnte man dazu neigen, sie als eine Form der Selbstskandalisierung zu betrachten. Ronald Hitzler $(1987,1989)$ hat bereits auf mögliche Gewinne der Skandalisierten hingewiesen, und im Zuge einer Theorie der "intendierten Selbstskandalisierung" (Haller 2013) wird angenommen, dass Prominente durch gezielte Provokationen und Normverletzungen einen Skandal initiieren in der aufmerksamkeitsökonomischen Absicht, einen Zugewinn an symbolischem und/oder realem Kapital zu erzielen. Die bei Haller aufgeführten Typen des "Skandalpolitikers" (ebd.: 156) oder auch des "Reality TV" (ebd.: 158) oder andere Motivationen zur Selbstskandalisierung scheinen jedoch auf Lewitscharoff wenig anwendbar. Auch auf eine strategische Fraktionierung, die eine erfolgreiche Skandalisierung auszeichnet, scheint Lewitscharoff keine Rücksicht genommen zu haben, wenn man sich etwa die negativen Stellungnahmen der Kirchen vor Augen hält. Auch sprechen die vielen metasprachlichen Selbstkommentare und die biographische Bezugnahme in der Rede nicht für diese Einordnung. Wenn vieles gegen eine intendierte Selbstskandalisierung spricht, so könnte es sich doch um eine in-Kauf-genommene Selbstskandalisierung handeln, die mit der in der Literatur prominenten Geste der "großen Anklage", also eines "J'accuse" (Émile Zola), auftritt. Dass die bloße Betrachtung des Skandalisierungsaspekts jedoch nur einen kleinen Teil der dadurch ausgelösten Prozesse beschreiben kann, soll im Verlauf der weiteren Analyse der Rede gezeigt werden.

\subsubsection{Die Dresdner Rede als Normverletzung}

Die Rede Lewitscharoffs (2014) besteht aus zwei Teilen, einem biografischen Teil, in dem es um Tod, Sterben und Glauben in ihrer Ursprungsfamilie geht und einem programmatischen Teil, der eine emotional stark aufgeladene "Abrechnung" mit der Kultur der Gegenwart beinhaltet. Nahtstelle zwischen diesen beiden Teilen ist ein Glaubensaxiom, ja ein Bekenntnis, das durch die vorangehenden biografischen Erzählungen über ihre gläubige, verehrte Großmutter und ihre ungläubigen, verachteten Eltern vorbereitet wird:

Mir kommt eher die Vorstellung, dass ich Herrin über mein Schicksal wäre, reichlich absurd vor. Ganz einfach, mein Schicksal liegt in Gottes Hand und nicht in meinen Händen.

(Lewitscharoff 2014: 7)

Von dort aus entwickelt sie im zweiten Teil eine Kulturkritik der Selbstbestimmung, die sie jedoch nicht diskursiv im Sinne eines Gesprächsangebots ausführt, sondern mit großer Emotionalität, die mehrfach ganze Personengruppen abwertet, die sie "Egomanen" oder einfach "diese Leute" nennt. Bezeichnet werden damit nicht nur Reproduktionsmediziner, sondern auch Feministinnen, Liberale, homosexuelle Paare, Menschen, die sich ein Kind durch künst- 
liche Befruchtung wünschen, und - hier wird der Bezug zu ihren Eltern deutlich - "Ungläubige".

Um sich von diesen in Gesamtheit abzugrenzen, zieht Lewitscharoff den Ausdruck "Selbstermächtigung" heran, in einer spezifischen Bedeutung: ${ }^{5}$ Der Ausdruck "Selbstermächtigung" wird hier in zentraler Weise als Kampfbegriff verwendet, gegen eine Kultur der Machbarkeit und Selbstbestimmung, die für sie eine Hybris darstellt. ${ }^{6}$ Mehrfach führt sie Nazivergleiche an, wenn es um eine selbstbestimmte Schwangerschaft geht. Durchgängig werden zentrale christliche Begriffe wie Erbsünde, Teufel, Himmel und Hölle bekenntnishaft eingesetzt, der ganze zweite Teil des Textes ist mit emotional abwertenden Ausdrücken wie "Abscheu", "lächerlich", "Blähvorstellung", "abscheulich", "grauenerregend", "widerwärtig" oder "abartig" durchsetzt. Der zweite Teil ihrer Rede enthält schließlich den Kulminationspunkt, der in der anschließenden Welle der Empörung immer wieder zitiert werden wird, wenn sie nämlich Kindern, die aus künstlicher Befruchtung hervorgegangen sind, das Menschsein abspricht, und den Eltern und Medizinern dabei Nazi-Mentalität unterstellt:

Mit Verlaub, angesichts dieser Entwicklungen kommen mir die Kopulationsheime, welche die Nationalsozialisten einst eingerichtet haben, um blonde Frauen mit dem Samen von blonden blauäugigen SS-Männern zu versorgen, fast wie harmlose Übungsspiele vor. Ich übertreibe, das ist klar, übertreibe, weil mir das gegenwärtige Fortpflanzungsgemurkse derart widerwärtig erscheint, dass ich sogar geneigt bin, Kinder, die auf solch abartigen Wegen entstanden sind, als Halbwesen anzusehen. Nicht ganz echt sind sie in meinen Augen, sondern zweifelhafte Geschöpfe, halb Mensch, halb künstliches Weißnichtwas. Das ist gewiss ungerecht, weil es den Kindern etwas anlastet, wofür sie rein gar nichts können. Aber meine Abscheu ist in solchen Fällen stärker als die Vernunft.

(Lewitscharoff 2014: 12)

\subsubsection{Reaktionen in der Öffentlichkeit}

Die Reaktionen in der Öffentlichkeit waren und sind - bis auf wenige Ausnahmen - durchgehend abweisend. Die ersten Reaktionen finden sich in den führenden Printmedien und ihren Onlinevarianten, die wiederum auf Twitter gepostet werden. In Twitter lassen sich die Schwärme bekanntermaßen über Hashtags, hier also \#Lewitscharoff, organisieren. So konnten für die vorliegende Untersuchung leicht alle relevanten Tweets gesammelt werden. Die folgenden Aussagen beziehen sich auf eine Sammlung aller Tweets, die im Zeitraum vom 06.03.2014 bis zum 09.04.2014 erschienen sind, wobei der Fokus der Auswertung auf den ersten drei Tagen lag (vgl. Liebert 2014).

Es fällt auf, dass die Onlinevarianten der Printmedien (insb. Spiegel, FAZ, Welt, Zeit, SZ, FR, taz, Huffington Post, Cicero) die klassische Funktion des Agendasettings einnehmen. Sie befeuern die Twitter-Timeline mit markanten Artikeln, die in den Tweets weitergepostet und kommentiert werden.

\footnotetext{
5 Wie vorhin ausgeführt, ist es unschädlich, wenn theoretische Begriffe wie Demokratie, Recht, Kraft, Masse, u. a. sowohl im fachlichen wie im öffentlichen Diskurs verwendet werden, wenn die fachlichen Ausdrücke definiert bzw. terminologisiert wurden.

6 Damit stellt sie sich in die literaturwissenschaftliche Tradition der Verwendung des Ausdrucks "Selbstermächtigung" (vgl. Liebert 2015).
} 
Beispiele: ${ }^{7}$

G.C.@GC. 7. März

Guter Artikel über die Hackfresse Lewitscharoff und den konservativ-dummdeutschen Backlash http://www.spiegel.de/kultur/gesellschaft/sybille-lewitscharoff-sybille-bergs-gedanken-zurskandalrede-a-957412.html ...

s.@s. 7. März

Doch, man darf sagen, was man denkt, q.e.d. Und man darf die Folgen ertragen. Oder einfach mal die Fresse halten. http://www.faz.net/aktuell/feuilleton/buecher/autoren/sibyllelewitscharoff-im-gespraech-darf-ich-nicht-sagen-was-ich-denke-12835124.html ...

Auf diese Weise wurden verschiedene Kritikansätze an Lewitscharoffs Rede eingebracht und aufgegriffen: So wurde der Faschismusvorwurf gegenüber Lewitscharoff zuerst vom Spiegel eingebracht und erst danach in der Timeline von anderen aufgegriffen und weiter ausgebaut, ähnlich verhält es sich mit dem Sarrazin-Vergleich, der zuerst von SZ und FR markant eingebracht wurde. Gleiches gilt übrigens auch für die wenigen Beistandsartikel etwa in der Welt und in Cicero, nach denen entsprechende (geringe) unterstützende Aktivitäten im TwitterSchwarm stattfinden. Die geringe Zahl der Beistandsartikel erklärt sich aus dem Text von Sibylle Lewitscharoff selbst: Vorhin wurde die Genreunsicherheiten in Bezug auf Lewitscharoffs angesprochen, in Frage stand, ob es sich möglicherweise um einen Text in der Tradition der engagierten Literatur und der Geste der Anklage handeln könnte. Anklage und Kulturkritik sind jedoch nur die Folge. Die Performanz von Lewitscharoffs Rede stellt eine Art "Outing" dar, ein öffentliches Bekenntnis zum Christentum, in dem sich eine offensichtlich lange aufgestaute Aggressivität eine Schneise bricht und gegen eine scheinbar "hegemoniale" Kultur der Selbstbestimmung, letztlich auch gegen das gesamte Projekt der Aufklärung auftritt, und die dagegen den Glauben an Gott setzt und von diesem her das Gottgewollte vom NichtGottgewollten, der menschlichen Hybris nämlich, unterscheidet. Dieses Bekenntnis und seine daraus folgende Kulturkritik und Umwertung ist in seiner Reichweite so fundamental (und daher auch keine strategische Fraktionierung), dass sich selbst die katholische Kirche davon distanzieren musste. Dies deutet auf ein ganz anderes Genre hin, das hier aktiviert wird, nämlich das der prophetischen, apokalyptischen Warnung, also eines "Kehret um!" eines Jesaja oder eines Jeremia. Die Prophetenrede verfügt über eine ähnlich emotionale Wucht und ist rücksichtslos (auch gegenüber religiösen Institutionen). Dadurch gehören nun allerdings nahezu alle Kulturangehörigen zum Kreis der Kritisierten. Bei der Bandbreite an Kritisierten verwundert es auch nicht, wenn im Folgenden kaum Unterstützung erfolgt, sondern ein großes negatives Echo hörbar wird und erste Ansätze von Status-Degradierungszeremonien zu verzeichnen sind.

\subsubsection{Zeremonien der Status-Degradierung von Sibylle Lewitscharoff}

Es gab verschiedene Ansätze für Status-Degradierungszeremonien in Bezug auf Sibylle Lewitscharoff, man findet sie bereits in der untersuchten Twitter-Timeline, wenn etwa versucht wird, ihr ihre literarische Kompetenz abzusprechen oder ihr Unwürdigkeit im moralischen Sinn vorwirft, da sie Menschen verachte. Weiterhin finden sich Wertungen, die sie als "geisteskrank" oder "sexuelle Versagerin" abstempeln, oder Nazi-, Faschismus- und Sarrazinver-

7 Um Klarnamen nicht rekonstruieren zu können, wurden die Twitternamen durch Initialen abgekürzt.

ISSN 1615-3014 
gleiche anstellen. Es gibt aber auch Versuche, sie für gebuchte Veranstaltungen wieder auszuladen oder ihr bereits erhaltene Ehren wieder abzuerkennen. Elemente einer StatusDegradierungszeremonie im Sinne Garfinkels finden sich auch über die Abwertung und Verhöhnung der körperliche Erscheinung von Sibylle Lewitscharoff, als Versuch, ihren "wahren Charakter" aufzudecken, dass sie nämlich nur aufgrund ihrer eigenen Frustration, keine Kinder zu haben, gegen die Reproduktionsmedizin Stellung nehme und damit unglaubwürdig sei. Eine konkrete Status-Degradierungszeremonie wird jedoch erst über eine Online-Petition zur Aberkennung des Georg-Büchner-Preises eingeleitet, die nun behandelt werden soll.

\subsubsection{Die Online-Petition gegen Sibylle Lewitscharoff}

Die Online-Petition "Aberkennung des Georg-Büchner-Kulturpreises für die Autorin Sybille Lewitscharoff" ${ }^{8}$ wurde von einer einzelnen Person am 07.03.2014 auf der Online-Plattform Openpetition.de in Gang gesetzt, nämlich von Cornelia Csuk (2014).

Der Blick auf die Daten in dem erhobenen Twitter-Korpus (Liebert 2014) zeigt, dass das Thema des Büchnerpreises unabhängig von der Petition eine Rolle gespielt hat:

M.@S.7. März

Der Georg-Büchner-Preis wird unbenannt - in Vollidioten-Pokal ... http://www.spiegel.de/kultur/literatur/sibylle-lewitscharoff-verteidigt-thesen-zuretortenkindern-a-957372.html\# $\mathrm{ref}=\mathrm{rss}$...

(Liebert 2014)

\section{H.G.@H. 8. März}

Wer hier in Deutschland so alles einen Büchner-Preis bekommt, macht mir Angst: http://www.3sat.de/mediathek/?mode=play\&obj=42137 ... \#Lewitscharoff

Wer von der Existenz der Online-Petition zur Aberkennung des Büchnerpreises erfahren hat, tut dies kund und wirbt um Unterstützung:

S.W.@SW: 8. März

Empfehlung! Petition zur Aberkennung des Georg-Büchner-Kulturpreises für die Autorin Sybille Lewitscharoff unter http://www.openpetition.de

M. E.@F.9. März

Unterschrieben: \#Petition Aberkennung des Georg-Büchner-Kulturpreises für die Autorin \#Deutschland http://bit.ly/1qfqcUo via@oPetition

Es zeigt sich hier eine Art Suchverhalten, was denn eine Statusdegradierung sein könne. Und in der Diskussion über Formen der Distanzierung werden auch Ehrungen in den Blick genommen, die wieder rückgängig gemacht werden könnten. ${ }^{9}$

8 Der Vorname "Sibylle" wird im Antragstext systematisch falsch geschrieben, nämlich als "Sybille".

9 Hierzu gibt es eine Debatte an der Universität Kassel, an der Lewitscharoff die Grimm-Gastprofessur innehatte. Dies kann an dieser Stelle aber nicht weiter einbezogen werden (vgl. Universität Kassel 2014). 


\subsubsection{Die Autorin der Online-Petition}

Laut Selbstauskunft im Internet hat Frau Csuk im Jahr 2012 ein Kind verloren und wollte selbst noch ein Kind mit Hilfe der Reproduktionsmedizin bekommen (vgl. Csuk 2013a). Diese Erfahrungen hat sie zusammen mit weiteren Informationen 2013 in dem Buch "Leitfaden Reproduktionsmedizin: Wege zum Wunschkind" bei Amazon im Verlag Books on Demand veröffentlicht (vgl. Csuk 2013b). Die Rede Lewitscharoffs muss daher für sie sicherlich eine tiefe Kränkung gewesen sein. Im Rahmen einer Status-Degradierungszeremonie ist die Anklägerrolle nur mit einer Person mit moralisch integren Motiven zu besetzen. Dieses erfüllt Frau Csuk durch ihre Geschichte: Sie ist eine glaubwürdige Anklägerin.

Allerdings ist sie keine Expertin für Literaturwissenschaft, um die literarische Qualität von Lewitscharoffs Werk, das zentrale Kriterium für die Verleihung des Büchnerpreises, beurteilen zu können. Im Rahmen einer Mentalität der Selbstermächtigung erscheint dies jedoch nicht als ein "Problem", da die Kriterien der Preisvergabe ja öffentlich vorliegen und nun von allen Bürgern "angewendet" werden können. Dem institutionalisierten Findungsprozess einer Gruppe von Experten zur Selektion im Rahmen eines Feldes literarischer Werke wird hier also die Eigenkompetenz durch individuelles Lesen der Statuten der Preisvergabe entgegengesetzt. So kann sie als Anklägerin auftreten und sich gegen die Experten der Darmstädter Akademie für Sprache und Dichtung mit Bezug auf die Büchnerpreis-Satzung positionieren. Es werden hierbei somit sämtliche institutionelle, reguläre Verfahren ignoriert und die eigene Kompetenz als gleichwertig gegen die wissenschaftliche Kompetenz der Akademie stellt. Dies wird im Antragstext deutlich:

Aberkennung des Georg-Büchner-Kulturpreises für die Autorin Sybille Lewitscharoff wegen unwürdiger Aussagen gegen kinderlose, schwule und lesbische Paare. Die Satzung für den Büchner-Preis beinhaltet folgenden Paragraphen (in der Fassung vom 21. März 1958): "Zur Verleihung können Schriftsteller und Dichter vorgeschlagen werden, die in deutscher Sprache schreiben, durch ihre Arbeiten und Werke in besonderem Maße hervortreten und die an der Gestaltung des gegenwärtigen deutschen Kulturlebens wesentlichen Anteil haben" Die Aussagen der Preisträgerin in der Öffentlichkeit sind geeignet, das Ansehen homosexueller Paare sowie Menschen, die auf medizinische Hilfen der Reproduktionsmedizin zurückgreifen müssen, nicht nur zu schädigen, sondern es gleicht einer Art Patienten-Bashing, das so keinesfalls unwidersprochen stehen bleiben darf. Frau Sybille Lewitscharoff hat dadurch bewiesen, dass sie dieser Ehrung absolut nicht würdig ist, denn nicht nur die Form und die Anwendung von Sprache in eloquenter Art und Weise, sondern gerade der Inhalt und das transportierte moralische Gedankengut sind ein Bewertungsmassstab, ob ein Autor einer Ehre würdig ist oder nicht.

Begründung:

Die Petenten fordern von der Deutschen Akademie für Sprache und Dichtung

Glückert-Haus

Alexandraweg 23

D-64287 Darmstadt

die verleihende Jury über die Aussagen der Autorin in Kenntnis zu setzen und eine Aberkennung des Kulturpreises zu initiieren.

Zu den Gründen hier einige Pressestimmen:

(Csuk 2014) 
Es folgt nun ein Link zu einem taz-Artikel und die Wiedergabe eines "offenen Briefes" von Robert Koall, zuerst Gastgeber Sibylle Lewitscharoffs, dann erster prominenter Kritiker ihrer "Dresdner Rede".

Bemerkenswert ist hier die quasi-juristische Argumentation mit Bezug auf die Satzung. Der Satzungstext wird von Frau Csuk nun in einer Weise interpretiert, die weit in das "Hoheitsgebiet" der Akademie und der Auswahlkommission eingreift:

denn nicht nur die Form und die Anwendung von Sprache in eloquenter Art und Weise, sondern gerade der Inhalt und das transportierte moralische Gedankengut sind ein Bewertungsmassstab, ob ein Autor einer Ehre würdig ist oder nicht.

(ebd.)

Bei Openpetition gibt es für jede Online-Petition eine Unterabteilung "Debatte". Dieser Debattenteil ist in die Bereiche "pro" und "contra" eingeteilt, in denen NutzerInnen ihre Meinung anonym publizieren können. Auch in der "Debatte" wird in großen Teilen juristisch argumentiert, dieses Mal mit dem Versuch, eine "echte Straftat" zu konstruieren:

Das ist eine Diffamierung in einem Ausmaß, dass [sic!] die Prüfung auf den Tatbestand der Volksverhetzung nahelegt.

$$
\text { (ebd., pro) }
$$

Diese wird dann hin und her debattiert. Fest steht allerdings: Niemand hat die Absicht, Lewitscharoff wegen Volksverhetzung vor Gericht anzuklagen, es handelt sich vielmehr um eine Laien-Rechtsprechung, deren Urteil und Urteilsvollzug selbst festgelegt werden.

Dabei entspringt die Macht für diese Status-Degradierungszeremonie einem Schwarm, bei dem auch bislang unpolitische Netze wie das "Klein-Putz.net", ein Forum für Menschen mit Kinderwunsch, politisiert ${ }^{10}$ werden, so dass die Zielzahl von 1000 problemlos erreicht wird. Abbildung 1 zeigt das durch und durch unpolitische Netzwerk und den Ersteintrag zur Debatte um Sibylle Lewitscharoff.

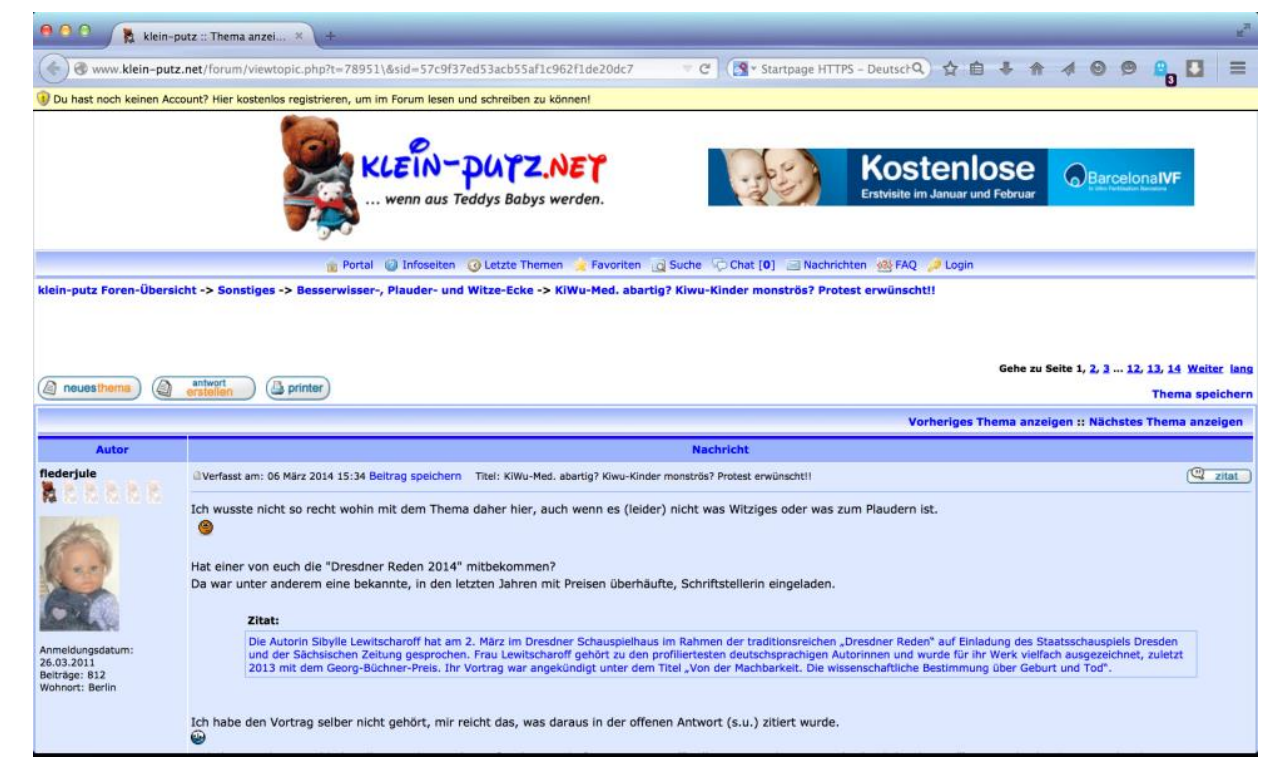

Abbildung 1: Das Kinderwunsch-Netzwerk "Klein-Putz.Net" www.klein-putz.net/forum /viewtopic.php?t=78951 $\backslash \&$ sid=57c9f37ed53acb55af1 c962f1 de20dc7 [23.02.2015]

10 Alle hier zitierten Beiträge sind ohne Anmeldung allgemein-öffentlich sichtbar. 
"Flederjule" schreibt dort:

Ich wusste nicht so recht wohin mit dem Thema daher hier, auch wenn es (leider) nicht was Witziges oder was zum Plaudern ist. Hat einer von euch die "Dresdner Reden 2014" mitbekommen? Da war unter anderem eine bekannte, in den letzten Jahren mit Preisen überhäufte, Schriftstellerin eingeladen. (...) Ich habe den Vortrag selber nicht gehört, mir reicht das, was daraus in der offenen Antwort (s. u.) zitiert wurde.

(Flederjule 2014)

Und sie endet in dem Aufruf:

Vielleicht hat ja jemand noch eine Idee was man öffentlichkeitswirksam machen könnte, meine Ideen wären erst mal: Boykottiert die Werke dieser Frau, schreibt ihr deutlich was ihr von ihrer Einstellung haltet und schreibt Protestbriefe an ihren Verlag, an Zeitungen und an die Institute bei denen sie Preise bekommen hat.

(Flederjule 2014)

Dies wird dann aufgegriffen und dortselbst auch bereits thematisiert:

Falls es eine Unterschriftenaktion für die Aberkennung des Georg-Büchner-Preises geben würde, wäre ich dabei. Andere mussten für solche Hassreden sogar Ämter beim Bund abgeben. Da wäre es nur richtig, dieser menschenverachtenden Rede Konsequenzen folgen zu lassen.

(Katharinchen 2014)

Dabei geht es bei der Aberkennung nicht um die literarische Qualität, für die Lewitscharoff den Preis erhalten hatte, sondern um die Beleidigung bzw. Verletzung, die durch die Rede erfolgt ist, und die nun gesühnt werden soll.

Im Beitrag von "Mondschaf" finden sich komprimiert die in der Twitter-Timeline (Liebert 2014) schon verstreut getweeteten Versuche, die moralische Integrität von Sibylle Lewitscharoff zu zerstören und ihren "wahren Charakter" zu entlarven, nämlich als "geisteskrank", "dumm", "Nazi", "nur materielle Interessen verfolgend" oder eine Kombination daraus:

ich neige ja dazu, leute mehr ernst zu nehmen und argumentiere sehr ungern und selten "unterhalb der gürtellinie". hier fällt mir aber nichts anderes ein. zumal ich mich als mutter eines (wunderbaren) "halbwesens" auch persönlich beleidigt fühle.

aber die frau ist ja einfach nur krank. sie würde doch so ein angebliches "halbwesen" auf der straße nicht erkennen können. ich kann absolut verstehen, wenn man sich persönlich gegen künstliche befruchtung entscheidet. aber wie kann man nur so dumm wie diese dame sein? das gute daran ist, dass die rede so bescheuert ist, dass das wohl nicht ernsthaft jemanden überzeugen wird.

oder es ist strategie, sie möchte wie sarrazin \& co durch provokationen von rechtsaußen die bei einer lyrikerin vermutlich eher dünnen auflagen steigern??

(Mondschaf 2014)

Wenn Lewitscharoff also "in Wahrheit" "geisteskrank" wäre oder "dumm" oder "ein Nazi" oder eine "Rechtspopulistin" oder "nur des Geldes wegen" Menschen mit Kinderwunsch beleidigte, dann wäre ihr Charakter als verderbt entlarvt. Als dann bekannt wurde, dass es die besagte Online-Petition gab, gab es reihenweise Mitteilungen über deren Unterzeichnung sowie Aufforderungen, dies ebenfalls zu tun: Das Netzwerk identifiziert sich zu einem "wir":

auch ich bin fassungslos. Aber wir sind ja nicht wenige, daher sollten alle die Petition unterschreiben. Aber es gibt noch mehr im Zeitalter der Onlineshops. Wo immer ihr Bücher findet, kann die geschmacklose Rede als Rezession hinterlassen werden. Die werde nämlich von sehr 
vielen gelesen. Das dürfte ihr Auftreten / Ansehen am besten bei ihren Anhängern verdeutlichen.

(Butscher 2014)

Das "Klein-Putz.net" synchronisiert sich und formiert einer politische Aktion: Es nimmt Teil an der Status-Degradierungszeremonie zur Aberkennung des Büchnerpreises von Sibylle Lewitscharoff, die von Cornelia Csuk ins Leben gerufen wurde.

Nun sind 1000 Menschen viel, wenn man sie sich gemeinsam auf der Straße oder einem Gerichtssaal vorstellt. Dennoch ist dies Anzahl der Unterzeichner im Vergleich zu anderen noch relativ gering. Im nächsten Fall soll eine Online-Petition mit mehr als 200.000 Unterzeichnern zu Sprache kommen, die versucht, eine deutlich schärfere Status-Degradierung zu realisieren.

\subsection{Zeremonien der Status-Degradierung von Markus Lanz}

\subsubsection{Die Moderation von Markus Lanz als Normverletzung}

Anlass für die bekannteste Status-Degradierungszeremonie gegen Markus Lanz war eine ZDF-Talkshow am 16.01.2014 mit den Hauptprotagonisten Markus Lanz, Hans-Ulrich Jörges und Sahra Wagenknecht.

Markus Lanz ist ein bekannter Moderator im Zweiten Deutschen Fernsehen (ZDF), der seit 2008 die regelmäßige Abendtalk-Sendung "Markus Lanz" moderiert. Lanz ist berüchtigt für einen insistierenden Fragestil, der den Gästen möglichst "Klartext" entlocken soll. Über diesen Moderationsstil gab es immer wieder Diskussionen, Markus Lanz stand darüber hinaus bereits seit Übernahme der ZDF-Traditionssendung "Wetten, dass..?" im Oktober 2012 in außergewöhnlichem Ausmaß in der öffentlichen Aufmerksamkeit, nicht nur, weil Moderatoren dieser Sendung generell stark im Fokus der Berichterstattung stehen, sondern auch, weil in diesem Sendeformat der Moderationsstil von Markus Lanz ebenfalls von Anfang an umstritten war.

Die Sendung "Markus Lanz" vom 16.1.2014 war jedoch außergewöhnlich, sowohl in Bezug auf die Schärfe der Moderation, als auch auf die Reaktionen in der Öffentlichkeit. Die bereits erwähnten Gäste in der Sendung "Markus Lanz" vom 16.1.2014 waren u. a. Sahra Wagenknecht, zu diesem Zeitpunkt Mitglied des Bundestages und erste stellvertretende Vorsitzende der Partei "Die Linke." und Hans-Ulrich Jörges, Mitglied der Chefredaktion des Wochenmagazins "Stern". In der Sendung, die mehrfach auf YouTube dokumentiert ist, griffen Lanz und Jörges in teils aggressiver Art die Position von Frau Wagenknecht an, versuchten, die "Linke" als verfassungsuntreu zu "entlarven". Ein gemeinsames, sich gegenseitig unterstützendes Vorgehen von Lanz und Jörges ist auch bei einer Betrachtung mit zeitlichem Abstand deutlich erkennbar, etwas spekulativer interpretiert, ging ein "Brüderclan" zu Werke: Frau Wagenknecht wurde regelrecht unter eine Art "Dauerfeuer" genommen, sie wurde sehr häufig unterbrochen, konnte kaum einen Satz zu Ende bringen, auch wurden unangemessene Fragen gestellt (bspw. Lanzens Frage nach Wagenknechts Gehalt als frühere Europaabgeordnete). Lanz hatte in diesem Gespräch die Kontrolle über seine Moderatorenrolle verloren bzw. diese nicht professionell wahrgenommen. Dies könnte man im Rahmen einer Verlaufsanalyse im Einzelnen zeigen, soll hier aber aufgrund des Beitragsumfangs nicht erfolgen. 


\subsubsection{Die Online-Petition gegen Markus Lanz}

Die Online-Petition "Raus mit Markus Lanz aus meinem Rundfunkbeitrag!" (Müller 2014) wurde von einer damals in der Öffentlichkeit unbekannten Frau, Maren Müller, auf der Openpetition-Plattform noch in der Nacht der Sendung publiziert (vgl. Radio Blau 2014). Sie zählte am Ende der ca. zweiwöchigen Laufzeit 233355 Unterstützer. Entsprechend reichhaltig war auch die Pro-/Contra-Kommentierung im Debattenteil der Petition.

\subsubsection{Der Text der Online-Petition}

Betrachtet man den Text der Online-Petition, so kommen bereits im Titel "Raus mit Markus Lanz aus meinem Rundfunkbeitrag!", aber auch in der weiteren Argumentation der Antragstellerin und noch deutlicher in den Kommentaren der vielen Unterzeichner die Grundzüge einer selbstermächtigten Status-Degradierungszeremonie zum Vorschein.

"Ankläger" und "Zeugen" treten hier in einem Akt der selbstermächtigten Repräsentation als Gebührenzahler und damit als die "eigentlich Befugten" über die Entscheidung auf, wer beim ZDF eingestellt wird und wer nicht. Als "Anklage" wird dabei folgendes vorgebracht:

Die Sendung "Markus Lanz" vom 16.01.2014 www.youtube.com/watch?v=pZdxHPWVm9M zeigte zum wiederholten Mal, dass Herr Lanz weder fähig noch willens ist, seinen Gästen gleichberechtigt Wohlwollen, Rederecht und Anstand entgegenzubringen. Ein Moderator, der offenbar große Probleme damit hat, dem politischen Spektrum links von der Mitte mit einem Mindestmaß an Höflichkeit zu begegnen, passt nicht in ein öffentlich rechtliches Format. Der im expliziten Fall miserable Stil im Umgang mit Sahra Wagenknecht spiegelt in drastischem Maße wider, dass politische Neutralität für Lanz ein Fremdwort ist.

Der Bildungsauftrag sowie die Wahrung der politischen und wirtschaftlichen Unabhängigkeit werden durch die von Lanz demonstrierte tendenziöse Diskussionskultur grob vernachlässigt.

Begründung:

Unzählige empörte Tweets zeigten bereits während der Sendung, dass viele Zuschauer es leid sind, von einem notorisch peinlichen Moderator durch diverse Sendeformate im Öffentlich Rechtlichen Rundfunk (in dem Falle ZDF) geführt zu werden. twitter.com $/$ search? $q=\% 23$ lanz\&src $=$ tren

Ein Moderator, der nicht fähig ist ohne Entgleisungen zu moderieren, den Offenheit und der Umgang mit abweichenden Meinungen offenbar überfordern, der Fragen stellt und die Antworten nicht hören will und der seine eigene Meinung stets über die seiner Gäste stellt, sollte nicht vom Beitragszahler alimentiert werden.

Ich fordere das ZDF daher auf sich von Markus Lanz zu trennen.

(Müller 2014)

Hier wird der ZDF-Moderator Markus Lanz "entlarvt" als einer, der in seinem Beruf der Moderation "inkompetent" sei, und darüber hinaus ein "moralisch verwerfliches" Gesprächsverhalten an den Tag lege. Dazu zählt, dass er Gesprächspartner aus "dem politischen Spektrum links von der Mitte" systematisch benachteilige.

"Inkompetenz" und "moralische Verwerflichkeit" zeigten in dieser Sicht also den "wahren Markus Lanz". Dessen Einseitigkeit verhindere, dass das ZDF unabhängig sei. Daher zeige die Normverletzung Lanzens nun vielmehr auf die Institution und deren Normverletzung, nämlich die "Unabhängigkeit" des ZDF; dessen "Bildungsauftrag" werde dadurch "grob vernachlässigt". Es wird hier also nicht nur gegen Lanz, sondern auch gegen das ZDF eine Sta- 
tus-Degradierungszeremonie eingeleitet; in vielen Kommentaren auch noch umfassender gegen den öffentlich-rechtlichen Rundfunk als Institution der deutschen Demokratie (vgl. dazu auch Bundeszentrale für politische Bildung 2009).

Dies kommt in folgendem Zitat zum Ausdruck:

Es geht doch garnicht vordergründig um Lanz! Es geht vor allem um die politische Kultur in solchen Talkshows. Und das wir deren Verrohung auch noch bedingungslos alimentieren müssen. So verstehe ich diese Petition vor allem gegen die unsägliche Haushaltsabgabe. Von dieser so ein Schwachsinn finanziert wird, ohne, dass sich jemand bewußt davon verabschieden könnte. Dies ist ein Ausdruck von institutioneller Gewalt. Gegen welche hier zaghaft angestritten wird. Ich wünsche der Petition überwältigenden Erfolg, so dass sie ein erstes Zeichen dafür setzt, dass sich die Bürger unseres Landes wehren.

(ebd., pro)

Es geht also nicht nur um Markus Lanz, sondern darum, Einfluss und Kontrolle auf die Institution auszuüben, indem man sie zwingt, dem von diesem Schwarm gehassten Moderator zu kündigen.

Der Charakter dieser Online-Petition als selbstermächtigter Status-Degradierungszeremonie wurde vor allem in der "Debatte" thematisiert: Wie im letzten Abschnitt ausgeführt, sieht das Textformat von Openpetition immer eine Unterabteilung "Debatte" vor, eingeteilt in die Bereiche "pro" und "contra", in denen NutzerInnen ihre Meinung anonym publizieren können.

Ein wesentlicher Einwand der bei "contra" zeichnenden Nutzer bezieht sich auf die Aufforderung an das ZDF, Lanz zu kündigen, was als eine unzulässige populistische Aggression gegen einen Einzelnen gesehen wird:

ihr Wunsch Markus Lanz mittels einer offenen Onlinepetition loszuwerden ... ist meiner Meinung nach einfach nur unanständig. Sie und jeder Petitionsunterstützer - in dieser speziellen Petition - handelt unanständig, da alle Petitionsunterstützer wissentlich in Kauf nehmen, dass ein TV Sender unter Umständen einen laufenden Vertrag mit einem festangestellten oder freiberuflichen Mitarbeiter auflöst. Es mag durchaus sein, dass Sie und viele andere sich über den Interviewstil von Markus Lanz aufgeregt haben, aber das rechtfertigt in keinster Weise eine Treibjagt. Ihre Online Petition ist aber

(Müller 2014, Beitragsabbruch an dieser Stelle wie im Orig., W.-A. L.)

Darauf wird von einem "pro" direkt entgegnet:

ja klar soll lanz raus. ich wäre glücklich wenn er seinen job verliert. er wird garantiert nicht auf der straße landen. hallo? mich fragt ja keiner ob ich fernsehen möchte und die gebühren zahlen möchte. was soll ich denn sonst tun um meiner meinung ausdruck zu verleien? aus dem fenster schreien? einen leserbrief an die redaktion schreiben? das zdf nimmt ja auch wissentlich in kauf dass ich mit so einer niveulosen scheisse belastet werde. ich will es nicht. mir unverständlich dass jetzt leute kommen und lanz verteidigen. ihr steht wohl so tief im wasser das ihr das ufer nicht mehr seht.

(Müller 2014)

Ein anderer "pro" macht, wie oben bereits angedeutet, im nächsten Zitat deutlich, dass es eigentlich um den Angriff auf die Institution ZDF bzw. des öffentlich-rechtlichen Rundfunks handelt. Der Schaden eines Einzelnen ist in dieser Sichtweise moralisch gerechtfertigt:

Die "Steinigung/Hexenjagd/usw." der Privatperson Markus Lanz ist eventuell verwerflich, allerdings gibt es eben auch die Sendung mit selbem Namen, gegen die zu protestieren legitim ist (es ist natürlich äußerst bedauerlich, dass die Privatperson dabei selbst betroffen ist) 
(Müller 2014)

Bei den meisten Unterstützern der Petition zeigt sich, dass sie keinerlei Bedenken haben, wenn ihre Aufforderung an das ZDF, Markus Lanz zu entlassen, auch tatsächlich nachgekommen werden würde. Dies wird wie eben gezeigt mit Aussagen gerechtfertigt, dass er schon wieder Arbeit finden werde oder einfach mit der Rechtfertigung, er habe ja genug Geld.

Während bei Lewitscharoff eine kleinere Degradierung, nämlich die Aberkennung des Büchnerpreises, geplant war, war die Sprache bei der Online-Petition gegen Markus Lanz deutlich aggressiver, das "Strafmaß" deutlich höher und entspricht dem, was Garfinkel unter einem geforderten Identitätswechsel versteht: Hier wurde die öffentlich-rechtliche Institution ZDF aufgefordert, das "Urteil", Markus Lanz zu kündigen, zu "vollstrecken", d. h. ihn nicht nur aus dem ZDF zu entlassen, sondern ihn aus seinem Sozialgefüge tatsächlich auszustoßen.

Das Ergebnis ist bekannt: Markus Lanz sprach eine Entschuldigung aus, die Frau Wagenknecht annahm; das ZDF hat ihn nicht entlassen. Die Degradierungszeremonie ist im Sinne des selbst gesteckten Zieles gescheitert. Dennoch bedeutete sie für Markus Lanz natürlich einen immensen Imageverlust und daher eine Status-Degradierung im weiteren Sinne (vgl. Deppermann 2005).

Aber auch der Angriff auf das ZDF als Institution hatte Folgen, nämlich den von der Initiatorin der Petition, Frau Müller, betriebenen Aufbau einer "Parallelinstitution", nämlich einer eigenen "Kontroll-Institution" in Form eines Vereins, die "Ständige Publikumskonferenz der öffentlich-rechtlichen Medien e. V." (2014), der zuletzt als Akteur in der Kritik an der Berichterstattung über angebliche "antirussische Tendenzen" in der Ukraine-Berichterstattung der öffentlich-rechtlichen Sender aktiv geworden ist (vgl. Bidder 2014).

\subsection{Selbstermächtigte Status-Degradierungszeremonien gegen Wissenschaftler}

Bei der Behandlung der Lewitscharoff-Debatte wurde bereits am Rande auf die Infragestellung der Kompetenz von Wissenschaftlern eingegangen, in diesem Fall die Infragestellung der LiteraturwissenschaftlerInnen der Darmstädter Akademie für Sprache und Dichtung, die das literarische Werk Lewitscharoffs mit dem Büchnerpreis geehrt hatte. Die Person, die in ihrem Status degradiert werden sollte, war jedoch keine Wissenschaftlerin, sondern Literatin.

WissenschaftlerInnen sind jedoch durchaus auch selbst Ziel selbstermächtigter StatusDegradierungszeremonien. Dies soll nun anhand der beiden Wissenschaftler Prof. Dr. Elisabeth Tuider und Prof. Dr. Andreas Kreiter gezeigt werden. Professor Tuider leitet das Fachgebiet "Soziologie der Diversität" an der Universität Kassel, Professor Kreiter ist Neurowissenschaftler an der Universität Bremen.

Die Normüberschreitung von Professor Kreiter war sein Einsatz von Tierversuchen mit Affen, Professor Tuider wurde angegriffen, weil sie eine freizügige Sexualaufklärung im Rahmen einer auf Vielfalt ausgerichteten Sexualpädagogik öffentlich vertrat bzw. vertritt. Obwohl beide Fälle Verschiedenheiten aufweisen, sowohl was die Thematik, als auch den Ablauf betrifft, handelt es sich immer um selbstermächtigte Status-Degradierungszeremonien, die auch die Institution Wissenschaft betreffen. Betrachtet man die Rolle der "Ankläger", so tritt gegen Professor Kreiter der Verein "Tierversuchsgegner Bundesrepublik Deutschland e. V." auf, bei Frau Tuider der Autor Akif Pirinçci, bekannt durch seinen Roman "Feldiae" und jüngst durch 
seinen emotionalen Ausbruch in seinem Pamphlet "Deutschland von Sinnen". Beide gehen entsprechend einer Status-Degradierungszeremonie vor, indem sie versuchen, die "wahren Motive" bzw. den "wahren Charakter" der angegriffenen Person "aufzudecken".

Im Folgenden soll die selbstermächtigte Status-Degradierungszeremonie gegen Professor Kreiter näher betrachtet werden.

Der selbstermächtigten Status-Degradierungszeremonie gegen Professor Kreiter, um die es nun gleich gehen soll, ging ein jahrelanger Rechtsstreit voraus, der schließlich am 20.01.2014 höchstrichterlich so entschieden wurde, dass seine Tierexperimente rechtlich unbedenklich seien, und aufgrund der Freiheit von Forschung und Lehre auch von einer Behörde nicht eingeschränkt werden könnten, wie dies die kommunale Bremer Aufsichtsbehörde versucht hatte (vgl. BVerwG 2014). Bereits seit Ende der 90er Jahren hatte es verschiedene Aktionen einschließlich Morddrohungen gegen Professor Kreiter und seine Familie gegeben. Bei einer funktionierenden Institution-Individuum-Beziehung wäre nun mit dem letztinstanzlichen Urteil vielleicht nicht Zufriedenheit, aber doch Rechtsfrieden einkehrt, da die Sache institutionell endgültig entschieden wurde. Entscheidend für die Mentalität der Selbstermächtigung ist wie oben ausgeführt jedoch, dass das Ergebnis der Institution nicht akzeptiert wird, sondern ein eigenes "Verfahren" mit eigener (hier juristischer und wissenschaftlicher) "Kompetenz" angestrengt wird, mit dem Ziel der (bislang "zu Unrecht" nicht erfolgten) StatusDegradierung.

Die kündigte sich bereits kurz nach der Urteilsverkündung an, als etwa Wolfgang Apel, Vorsitzende des Bremer Tierschutzbundes und Ehrenpräsident des Deutschen Tierschutzbundes, in Radio Bremen sagte:

Was das Gericht sagt, interessiert mich in keinster Weise. Hier geht es um Lebewesen. Da werde ich weiter kämpfen. Ich sehe diese wissenschaftlichen Experimente an der Universität nach wie vor nicht als legal an.

(Apel 2014)

Schließlich wurde vom Verein "Tierversuchsgegner Bundesrepublik Deutschland e. V." eine Anzeigen-Kampagne gegen die Person von Professor Kreiter in Gang gesetzt: Am 16. und 17.4.2014 wurde eine ganzseitige Anzeige in verschiedenen überregionalen Tageszeitungen (u. a. FAZ, Zeit) veröffentlicht. ${ }^{11}$ Die besagte Anzeige wird auf der Homepage des Vereins nach wie vor (Stand 26.05.15) angeboten (Abbildung 2).

11 Wie schon vorher zu beobachten war, zeigen sich hier einige Verstärkungsmechansimen zwischen der Empörung in Kulturen der Selbstermächtigung und den traditionellen Medien, die hier aber nicht weiter verfolgt werden können. 


\section{KREITER}

\section{macht eiskalt weiter}

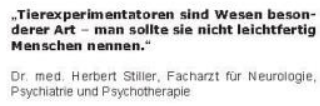

M
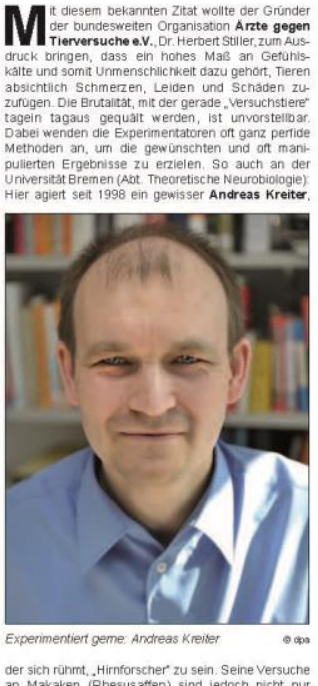

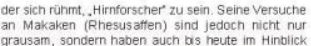

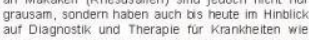

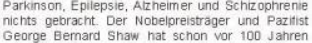

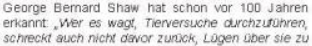

Leere Versprechungen

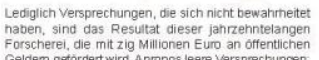
Geldem getordert wirr. Apropos leere versprecthungen
1998 hatte die Bremer

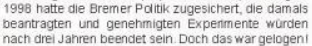

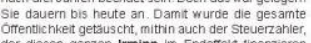
der diesen ganzen irrsinn im Enderent tinanzieren
muss. Immer wieder wurrde die Genentrigung zur

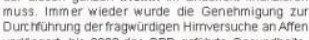
veriangert, bis 2008 das SPD-getunte Gesundheily
ressont endilich einen neuen Antrag aur Fortserturn

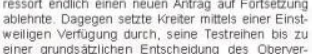

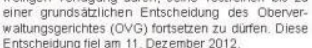

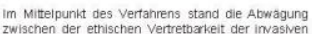

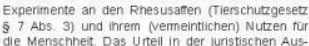
Bremen durfen cie Afren wether massaknert werden!
Auserdem wird der Genennmigungsbehórde jeglicher

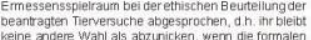
Voraussetzungen entintsind, so dieurteilsbegrindung

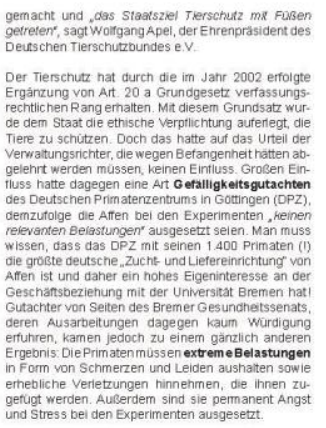

Leiden ohne Ende

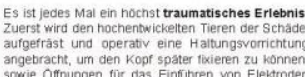

angebracht, um den Kopt spater tivieren zu konne
sowie Ofrnungen fur das Eirnuthren von Eleitrodode

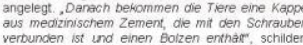

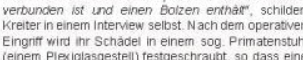

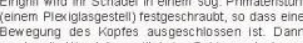

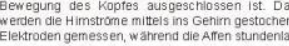

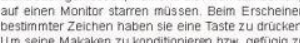

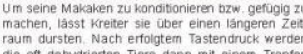

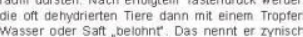

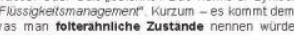
Die, cchronischen Versuche" lauten uber menres
Jahre, d.h. die Tilere sind uber einen sehr langen Ze: aum wâhrend inrer linbensianglichen Gerangensct Iimmer wieder diesen schreckichen Torturen aus
setzt. Hinzu kommt sett einiger Zet noch der Eins

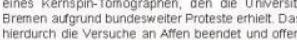

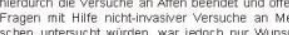
denken. Denn Kreiter settr das Gerz seitsem
seine Experimente (1) ein. Dabei nimmt er bilitige in Kaut, dass es durch den sehr hohen Schallpeg
Zu schiveren Horschaden bel den ungeschutze

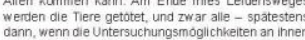

\section{Lizenz zum Töten}

Das Unteil des ovG stent einen Freibrief fur Ther
qualerei und eine Lizenz zum Toten der, Versuchstier:

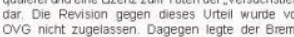

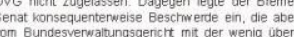
Keugenden Begrundung abgeschmetrett wurde, Und es bestunde kein weiterer raarungsbedart. sachverhat wurde von den Richterm so behandete tart nicht verwattet, sondem muss verboten werden, xperimente, da es sicht im vor Sinn und Zweecenden Fall - Neugiertorschung" othne die Notwendigket eines

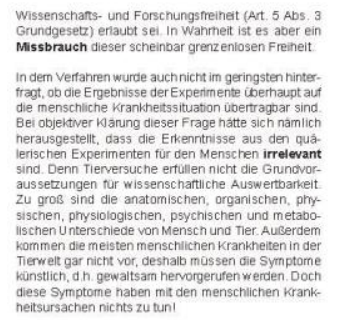

\section{Russisch Roulette}

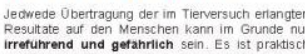

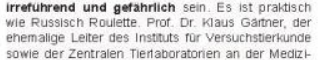

sowie der Zentralen Tienaboratorien an der Medizt
nischen Hochschule Hannover, hat es aut den Pun

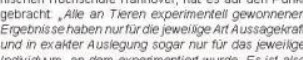

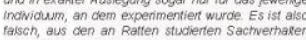
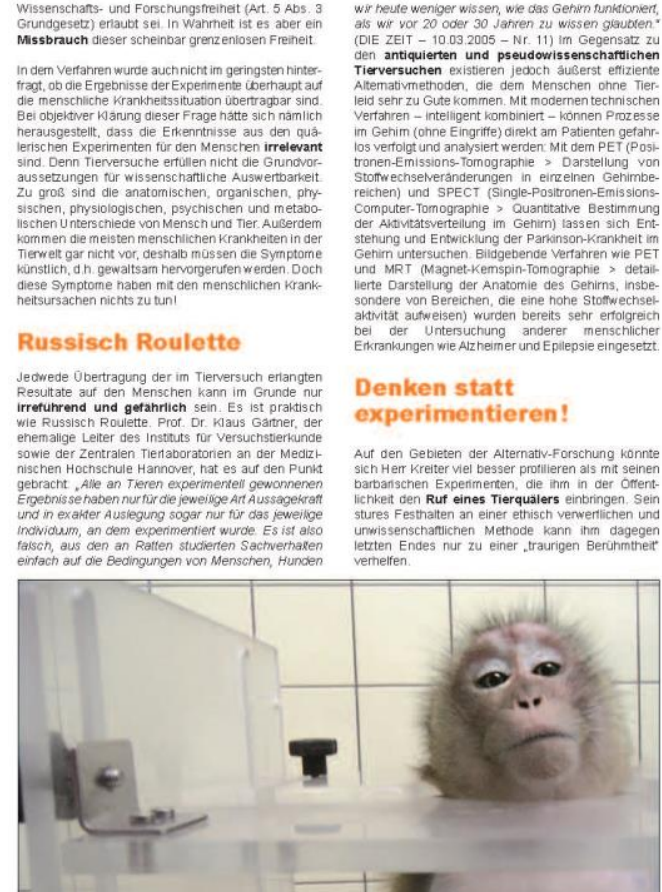

Denken statt

experimentieren?

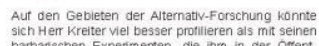

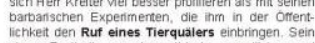
kann inthr dagegerer

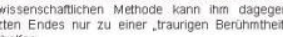

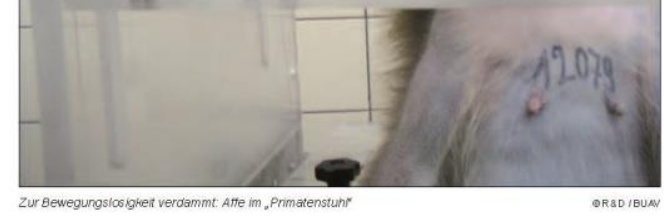

APPELL

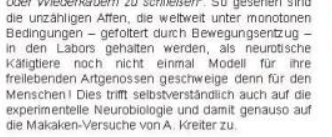

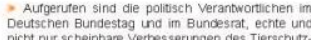
geseteres, das in der jetzigen Fassung nicht die opter,

Aufferuten ist der Reltor der Universittat Bremen,

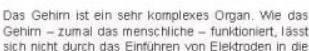
sich nicht durch das Eintuhren von Eleltroden in a
10 Milliarden silien heraustinden. Jede Stroring von ausen muss do des Hims und den Elektrodradraten werderen urvortherSenbare Kontakte nergestellt, die jegilches Resultal

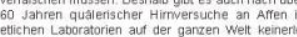

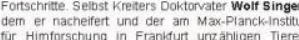
Locher in den Schadel gebonit hat, gibt in einet

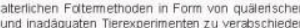

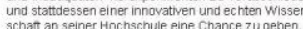

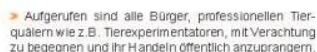
Leser mil starken Nerven kbonnen sich bel Youtube
einen Eintruck verschaffen, wie es in einer der gróis.

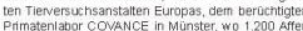
gehaten und gequat werden, zugent

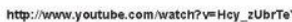

\section{TIERVERSUCHSGECNER BUNDESREPUBLK DEUISCHLAND EVV.}

列

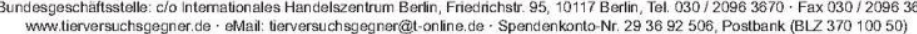

Abbildung 2: "Kreiter macht eiskalt weiter." Anzeigenkampagne des Vereins "Tierversuchsgegner Bundesrepublik Deutschland e. V." www.tierversuchsgegner.de/downloads/AZ-Kreiter-A3.pdf [05.03.2015]

In der Überschrift wird die Normverletzung klar genannt, dabei wird "Kreiter" auf "weiter" gereimt, was eine sozusagen bereits im Namen angelegte Uneinsichtigkeit suggeriert, hinzu- 
kommt, dass die Uneinsichtigkeit "eiskalt" sei, d. h. 'ohne Herz' und 'ohne Mitgefühl' für Tiere.

Dies wird in den ersten Absätzen der Anzeige dann auch entsprechend ausgeführt, indem zunächst auf der Ebene der Kategorien, die Kategorie der "Tierexperimentatoren" generell als nicht-menschlich und durch "Gefühlskälte", "Unmenschlichkeit" und "Brutalität", die "unvorstellbar" sei, gekennzeichnet wird. Darüber hinaus werden WissenschaftlerInnen, die Tierversuche einsetzen auch generell unter Manipulationsverdacht gesetzt, denn Angehörige der Kategorie "Tierexperimentatoren" wendeten "ganz perfide Methoden an, um die gewünschten und oft manipulierten Ergebnisse zu erzielen" (Gaertner 2014).

Dann wird die Person von Professor Kreiter als ein Element dieser Kategorie eingeführt, das somit die genannten Eigenschaften ("gefühlskalt", "unvorstellbar brutal", "wissenschaftlich unseriös") aufweist: "So auch an der Universität Bremen (Abt. Theoretische Neurobiologie)" (ebd.).

Die erste Charakterisierung von Professor Kreiter in diese Anzeige zeigt viele Merkmale der Selbstermächtigung: Die Missachtung seiner institutionellen Würde und Reputation als Professor einer deutschen Universität ("ein gewisser Andreas Kreiter"), das Forschen, das als bloßes "agieren" dargestellt wird, das Behaupten der Kompetenz, als Nichtwissenschaftler die wissenschaftlichen Leistungen von Professor Kreiter beurteilen zu können, das Aussprechen des Verdachts, er sei gar kein Forscher, sondern würde sich nur "rühmen", ein solcher zu sein. Schließlich gehört auch das Laienurteil über die Institution hier hinein, das lapidar behauptet, seine Forschungen hätten "nichts gebracht". Allein mit den ersten Abschnitten wird damit der "wahre Charakter"" von Professor Kreiter "entlarvt" als ein besonders "eiskalter" Vertreter der Zunft der "Tierexperimentatoren", die generell "gefühlskalt" und "brutal" sind, darüber hinaus "wissenschaftlich unseriös" vorgehen und auf Staatskosten ihren "Hang zur Brutalität und Tierquälerei" nachgehen. Durch diese "Anklage" ist bereits der erste Teil der selbstermächtigten Status-Degradierungszeremonie erfolgt.

Bevor nun der eigentliche Aufruf zur Ächtung von Professor Kreiter vorgetragen werden kann, muss zuerst das reguläre Gerichtsverfahren des Bundesverwaltungsgerichts, das ja das genaue Gegenteil von der eben aufgebauten Charakterskizze erbracht hat, als unrecht und ungültig dargestellt werden, was dann erst die Legitimität für weitere Aktionen mit dem Ziel der Status-Degradierung von Professor Kreiter ergibt:

Im Mittelpunkt des Verfahrens stand die Abwägung zwischen der ethischen Vertretbarkeit der invasiven Experimente an den Rhesusaffen (Tierschutzgesetz $§ 7$ Abs. 3) und ihrem (vermeintlichen) Nutzen für die Menschheit. Das Urteil in der juristischen Auseinandersetzung ist skandalös: An der Universität Bremen dürfen die Affen weiter massakriert werden! (...) Damit wird das Genehmigungsverfahren zur Farce gemacht und "das Staatsziel Tierschutz mit Füßen getreten", sagt Wolfgang Apel, der Ehrenpräsident des Deutschen Tierschutzbundes e. V. Der Tierschutz hat durch die im Jahr 2002 erfolgte Ergänzung von Art. 20 a Grundgesetz verfassungsrechtlichen Rang erhalten. Mit diesem Grundsatz wurde dem Staat die ethische Verpflichtung auferlegt, die Tiere zu schützen. Doch das hatte auf das Urteil der Verwaltungsrichter, die wegen Befangenheit hätten abgelehnt werden müssen, keinen Einfluss. Großen Einfluss hatte dagegen eine Art Gefälligkeitsgutachten des Deutschen Primatenzentrums in Göttingen (DPZ), demzufolge die Affen bei den Experimenten "keinen relevanten Belastungen" ausgesetzt seien. Man muss wissen, dass das DPZ mit seinen 1.400 Primaten (!) die größte deutsche "Zucht- und 
Liefereinrichtung" von Affen ist und daher ein hohes Eigeninteresse an der Geschäftsbeziehung mit der Universität Bremen hat!

(Gaertner 2014, im Orig. mit Hervorh.)

Ein Nutzen von Tierversuchen könne danach nur in einem "(vermeintlichen) Nutzen" bestehen, das Urteil sei "skandalös", mit den Affen würden keine Experimente durchgeführt, wie das Gericht feststellte, sondern diese würden vielmehr "massakriert werden", "Genehmigungsverfahren" würden "zur Farce gemacht", "das Staatsziel Tierschutz mit Füßen getreten". Weiterhin wird geurteilt, dass die Richter "wegen Befangenheit hätten abgelehnt werden müssen", sie ließen sich nicht von juristischen, sondern von "ökonomischen" Belangen leiten und hätten daher auch keine objektiven Gutachten einbezogen, sondern lediglich "Gefälligkeitsgutachten des Deutschen Primatenzentrums in Göttingen". Dem Bundesverwaltungsgericht wird also letztlich Bestechlichkeit vorgeworfen: "Bei objektiver Klärung dieser Frage hätte sich nämlich herausgestellt, dass die Erkenntnisse aus den quälerischen Experimenten für den Menschen irrelevant sind" (ebd.).

Nachdem das Urteil der Institution "entkräftet" wurde, erfolgt dann der "selbstermächtigte Strafvollzug": Nach einer zusammenfasenden, dramatisch noch einmal zugespitzten Reprise des Charakterbilds von Professor Kreiter als "Tierquäler" mit "barbarischen Experimenten", der "wissenschaftlich unseriös" und "unethisch" und "nur aus Ruhmsucht" arbeite, wird an die Öffentlichkeit (d. h. hier also u. a. an die LeserInnen der ZEIT, der FAZ usw.) appelliert, Tierquäler, als der Professor Kreiter gerade noch einmal ausgewiesen wurde, öffentlich zu schmähen und "öffentlich anzuprangern".

Aufgerufen sind alle Bürger, professionellen Tierquälern wie z. B. Tierexperimentatoren, mit Verachtung zu begegnen und ihr Handeln öffentlich anzuprangern.

(Gaertner 2014)

Selbst als es danach Proteste von Seiten wissenschaftlicher Institutionen wie z. B. vom Rektor der Bremer Universität Bernd Scholz-Reiter (2014) oder von der Allianz der Wissenschaftsorganisationen (2014) gab, die in ihrer Kritik auch die Medienakteure betrafen, die die Anzeige veröffentlicht hatten, rechtfertigte der Verantwortliche für die Anzeigenkampagne, der Vorsitzende der "Tierversuchsgegner Bundesrepublik Deutschland e. V." Rainer Gaertner, die Aktion:

Es muss in einer Demokratie erlaubt sein zu hinterfragen, ob jemand, der seit über 30 Jahren aus Neugier Tiere systematisch quält und tötet, nicht den Anspruch auf Menschenwürde verloren hat.

(Gaertner 2014 zit. nach Becker 2014)

Hier wird eine große Kluft zwischen verschiedenen Kulturen der Selbstermächtigung und demokratischen Institutionen sichtbar, aber auch gegenüber wissenschaftlichen Institutionen. Medienakteure spielen dabei eine durchaus schillernde Rolle, sie sind häufig Anfeuerer und Bühnengeber und können sich so gegenüber der "fünften Gewalt" positionieren. ${ }^{12}$

12 Traditionelle Medien profitieren von selbstermächtigten Status-Degradierungszeremonien, da sie sich gern als Kritiker der Institution inszenieren und dabei die in sozialen Medien häufig anzutreffenden, emotionalisierten Vereinfachungen wie gut - böse oder Täter - Opfer übernehmen, deren Emotionalität durch Schauerberichte des 
Ähnlich ist die selbstermächtigte Status-Degradierungszeremonie gegen Professor Tuider verlaufen. Frau Tuider wurde die Kompetenz als Wissenschaftlerin von Laien abgesprochen. Gerade Letzteres, das Absprechen der Kompetenz von Wissenschaftlern durch NichtWissenschaftler, weiter gefasst sogar das Absprechen von Wissenschaftlichkeit bestimmter Disziplinen, lässt sich noch häufiger finden. Professor Tuider sollte durch Pirinçcis Hassreden etwa eine "ins Perverse gehende" Persönlichkeitsstörung "nachgewiesen" werden, die nur deshalb zu einer Professur gekommen sei, da ein bereits institutionalisiertes "rot-grünes Netzwerk" in der Genderforschung eine Art "ideologisches Bollwerk" aufgebaut habe, das daher auch keine wissenschaftliche, wohl aber eine "ideologische Qualifikation" verlange. Zum Diskurs über Professor Tuider liegen ebenfalls Daten aus den Internet-Auftritten von Pirinçci und den darauf verweisenden Blogs vor, die zeigen, dass hier außer der Kompetenzabsprechung ein enormes Potenzial an sexualisierter Gewalt sichtbar wird, verfasst in einer teilweise extrem obszönen und gewalttätigen Sprache, auf deren Zitationen in diesem Zusammenhang verzichtet werden soll, auch wenn sie über einfache Suchabfragen jederzeit auffindbar und frei zugänglich sind. ${ }^{13}$ Vergleichbares haben etwa auch die \#AufschreiInitiatorinnen erfahren (vgl. dazu Strick 2013).

Beiden Fällen ist gemeinsam, dass das "Urteil" in einem Aufruf zur allgemeinen Ächtung, insbesondere zu einer Herabsetzung der Würde der betreffenden Person, besteht. Dabei wird in Kauf genommen, dass Einzelne mit Bezug auch diese selbstermächtigte StatusDegradierungszeremonie nun diese Ächtung auch tatsächlich durchführen, durch Morddrohungen, Schmähungen, oder auch Gewaltakte (vgl. Greiner/Demling 2014).

Beiden ist jedoch auch gemeinsam, dass sie in einem diskursiven Umfeld stattfinden, das bereits lange zuvor emotional aufgeladen war. Diese diskursive strukturbildende Vorbereitungsphase von selbstermächtigten Status-Degradierungszeremonien (im letzteren Fall der stark emotionalisierte Diskurs um Gender und Diversität) ist bislang noch wenig erforscht und müsste im Rahmen einer größeren Studie untersucht werden.

\subsection{Lynchen 2.0}

Das Ergebnis der eben besprochenen selbstermächtigten Status-Degradierungszeremonien war ein Aufruf zur öffentlichen Bloßstellung der "Verurteilten", ohne allerdings selbst zu konkreten Gewalthandlungen aufzurufen oder diese zu planen. Die Form dieser "Aufrufe" genügte allerdings, um auszulösen, dass sich Einzelpersonen selbst zu Bestrafungsaktionen wie anonymen Morddrohungen, Schmähungen usw. ermächtigten.

Eine weitere Forcierung liegt vor, wenn in der selbstermächtigten StatusDegradierungszeremonie die Strafaktion auch in Form (schwerer) Gewalttaten öffentlich geplant und deren Ausführung koordiniert wird. Dieser Typ von selbstermächtigter StatusDegradierungszeremonie entspricht dem Vorgang des "Lynchens" oder zumindest dem Spiel mit dem Lynchen, was nun exemplifiziert werden soll. Es handelt sich um den Fall eines jungen Mannes aus dem Westerwald, von dem ein Video in Umlauf gebracht wurde. Auf diesem

\footnotetext{
"Bösen" dann sogar noch gesteigert wird. Dies kann hier jedoch nur angedeutet werden, da dies durch größere Studien plausibilisiert werden müsste.

13 Zum Problem einer "Datenschau" bei einer Viktimisierung vgl. Pörksen und Detel 2012: 15-17.
} 
Video ist zu sehen, wie er seinen Hund anschreit, beschimpft, dabei packt und rüttelt und ihn mit Hassreden überzieht (vgl. SWR 2014). Das Besondere an diesem Fall ist, dass es sich hier nicht um einen Prominenten handelt, sondern um einen bis dahin völlig Unbekannten - es geht bei selbstermächtigten Status-Degradierungszeremonien also nicht einfach um die Lust am Zufallbringen von Prominenten. Die Qualität ist in diesem Fall auch insofern anders, als die "Ankläger" eine Akteurkoalition darstellen, nämlich mehrere (anonyme) Facebookseiten und eine große Menge daran beteiligter "User". Eine dieser Seiten soll nun näher betrachtet werden. Es handelt sich um die Seite, deren AutorIn den Namen "Jeder Tag ohne dich, ist eine Qual für mich" (2014a) trägt. Auf dieser Seite ist an der Stelle des Identifikationsbildes zwar das Bild einer jungen Frau, vorhanden, es handelt sich aber offensichtlich um einen Avatar, d. h. der/die Autor/in - und in diesem Fall der/die Ankläger/in - bleibt im Anonymen. Diese Facebookseite war eine zentrale Sammelstelle der selbstermächtigten StatusDegradierungszeremonie gegen den Westerwälder. Unter dem Blickwinkel einer Textsorteneinordnung handelt es sich zunächst einfach um eine Meinungsseite. Von diesen Meinungsseiten gibt es sehr viele. Meist werden auf diesen Seiten "originelle" Fragen zu Allerweltsthemen gestellt, die die Leser in persönlicher Weise beantworten sollen, eine Textsorte, die sich im Moment einer gewissen Beliebtheit erfreut. Diese Seiten haben aber noch einen anderen Zweck: Es soll viel "Traffic" erzeugt werden, viele Klicks, denn dann steigen Bekanntheit und ggf. Werbeeinnahmen. ${ }^{14}$

Der/die Autor/in dieser Facebookseite veröffentlichte nun unmittelbar nach Bekanntwerden des Falles und der ersten Forderungen nach Todesstrafe (vgl. SWR 2014) ein Bild des "Angeklagten" und "nominierte" ihn als "Hurensohn":

Heute, den 07.03.2014 nominiere ich ihn als Offizieller Hurensohn des Jahres! Wer nominiert mit?!

(s. n. 2014b)

In kurzer Zeit klickten 100.000 Personen auf den Like-Button "gefällt mir", Tausende posteten Kommentare. Es wurde die "Todesstrafe" für ihn gefordert, seine Privatadresse wurde veröffentlicht und Gewalttaten gegen ihn angekündigt. Auch wurden bereits Strafaktionen für seine Zeit im Gefängnis geplant, um ihn dort körperlich zu schädigen.

Gab es in den bisherigen selbstermächtigten Status-Degradierungszeremonien zumindest argumentativ-diskursive Ausführungen, so genügt in diesem Typ der Hinweis auf ein Video, einen in den traditionellen Medien geäußerten Verdacht, eine polizeiliche Vernehmung oder einfach die pure Behauptung, um eine selbstermächtigte Status-Degradierungszeremonie in Gang zu setzen. Auch hier fällt wieder auf, dass dies nicht bei allen Kategorien funktioniert. Denkt man etwa an normverletzende Fälle wie jemanden, der einer alten Dame die Handtasche stiehlt, oder auch an einen "normalen" Mord, dann käme nicht unbedingt eine solche Empörungswelle zustande. Es müssen also bereits in der diskursiven Vorgeschichte entstandene, emotional aufgeladene, verfestigte Charakterbilder wie die des Tierquälers bestehen, die dann durch eine einfache Identifikationsbehauptung wie Person A ist ein X (Tierquäler usw.) auf diese identifizierte Person projiziert werden und damit eine "Erlaubnis erteilt" wird, sämt-

14 Diese "Ökonomie der Empörung" war schon bei den traditionellen Medien sichtbar. 
liche Aggressionen, die sich als "gerechte Empörung" darstellen lassen, auszusprechen und ggf. auch auszuleben.

Gleichzeitig bildet sich allerdings auch ein Metadiskurs, in dem die selbstermächtigte StatusDegradierungszeremonie reflektiert wird. Dort wird mit Hinweis auf die Rechtssicherheit für alle Bürger zur Nachdenklichkeit und Besonnenheit aufgerufen. Auch in den vorhin diskutierten Beispielen ist aufgefallen, dass sich ein Bewusstsein manifestiert, dass durch selbstjustizähnliche Formen möglicherweise demokratisch legitimierte Institutionen gefährdet seien. Als Beispiel soll ein kommentierter Beitrag besprochen werden. Alle Zitate entstammen der oben angegebenen Quelle, die Zahl vor dem Datum ist die Zahl der so genannten "Likes", d. h. derjenigen Personen, die diesen Text gelesen und positiv bewertet haben. Um eine Identifizierung der Klarnamen zu erschweren, wurden die Beiträge initialisiert und mit einem Asterisk versehen.

H.*

Todesstrafe? Natürlich ist der Typ schwach gesalzen im Kopf und das was er macht ist grausam, aber Todesstrafe? Natürlich muss er bestraft werden, aber ihr habt kein Recht seinen Tod zu fordern. Ich bin froh, dass wir in einem Rechtsstaat leben, in dem Menschen anhand von Gesetzen verurteilt werden und nicht anhand von Meinungen in der Bevölkerung. $242 \cdot 07.03 .2014$ um 22:19

(s. n. 2014b)

Auf diesen Beitrag werden u. a. folgende Antworten gepostet:

R.*

Woher nimmst Du Dir denn das Recht mirluns zu sagen was meinlunser Recht ist? Wieso nimmst Du Dir das Recht hier etwas zu posten? Wir leben in einem Land in dem offiziell so etwas ähnliches wie Meinungsfreiheit herrscht! Wenn also jemand der Meinung ist dass ein gewisser Straftatbestand mit dem Tode bestraft werden sollte, dann ist es sein Menschenrecht diese Meinung zu äussern! Und ausgerechnet DU willst jemandem dieses Recht aberkennen!? 10 • 08.03.2014 um 00:19

(ebd.)

L.*

Du hast das recht zu sagen er hätte die todesstrafe verdient (was aber auch sschon greenzwertig is) bzw man sollte sie einführen...aber du hast kein recht sie zu fordern, also den richtern/gerichten zu sagen wie sie es machen müssen 3 - 08.03.2014 um 00:40

S.*

na toll, was für eine strafe gibt es denn dafür? richtig, eine geldstrafe... superl er ist in behandlung, das hat dem armen hund aber nicht in der situation geholfen! dem sollte man die arme und beine bei vollem bewustsein abhacken. so das er niemals mehr jemandem schaden kann. er sollte genauso leiden wie der hund oder andere tiere die er gequält hat! $11 \cdot 08.03 .2014$ um 01:04

(ebd.)

Im Folgenden wird bei einem weiteren Versuch, die Lynchstimmung auf dieser Seite Einhalt zu gebieten, zum einen wiederum mit dem Verweis auf das Recht auf Meinungsfreiheit, dann aber auch mit der moralischen Verpflichtung der Zivilcourage und des politischen Widerstands, argumentiert: 


\section{L.*}

Ich betone ausdrücklich, dass ich nicht mit $\mathrm{T}^{* 15}$ sympathisiere und seine Tat als höchst verwerflich ansehe. Das er eine gerechte Strafe verdient hat, das ist klar. Auf das Töten und/ oder Verletzten eines Wirbeltieres stehen in Deutschland 'nur' 3 Jahre Freiheitsstrafe oder eine Geldstrafe (dies geht aus dem Tierschutzgesetz kurz TierSchG, elfter Abschnitt, Straf- und Bußgeldvorschrifften $\S 17$ hervor). Aber hier rumzugrölen: 'Komm, den bringen wir um...', 'man sollte ihn erschiessen..' usw. ist nunmal genauso verwerflich und auch strafbar. Ich möchte an dieser Stelle noch einmal darauf hinweisen, dass ich in keinster Weise mit ihm sympathisiere, aber Seiten wie diese haben nun mal keinen Sinn (da 'Sammelklagen' in Deutschland keine Wertigkeit vor dem gericht haben) und da sie mit den extremen Mitläufern einher gehen. Ich möchte an dieser Stelle an euch appellieren, dass ihr solche Aussprüche wie: 'wir töten ihn' usw. unterlasst. 121 . 07.03.2014 um 14:57

(ebd.)

B.*

Deinen moral-apostolischen Hirnerguss kannst du am Sonntag in der Kirche von dir lassen und dich mit deinesgleichen austauschen. Nicht am Bösen geht die Welt zu Grunde, sondern an so uncouragierten Weicheiern wie dir, die zusehen, sich wie in deinem fall auf den Rechtstaat berufen - da wird mir schlecht. Die Seite gehört verbreitet, der gehört gebrandmarkt bis, dass ihn genug Leute kennen und er sich nicht mehr auf die Straße traut. 16 07.03.2014 um 15:15

$(\ldots)$

\section{L.M:*}

Guter Beitrag! Wenn wir alle mit Selbstjustiz anfangen, dann herrscht hier bald wieder das altbekannte Gesetz: der Mensch ist des Menschens Wolf...die Freiheit des Internets lässt zumindest erahnen, wie es dann hier aussehen würde! Natürlich muss diese...Mehr anzeigen 29 . 07.03.2014 um 16:06

(ebd.)

Zwei Dinge sind anders, als die bisher besprochenen Fälle: Der Angeklagte wird individualisiert und auffindbar gemacht, identifiziert, und es wird auf einer frei zugänglichen Internetseite öffentlich dazu aufgefordert, ihn körperlich anzugreifen und zu bestrafen. Zugleich setzt aber auch ein Diskurs ein, der versucht, die Lynchstimmung zu dämpfen.

Dieser Fall zeigt daher den Übergang von einer selbstermächtigten Status-Degradierungszeremonie zu einer im Netz organisierten Lynchjustiz, die teilweise auch in die Tat umgesetzt wird. Dies ist keine Einzelerscheinung: Am bekanntesten ist der Fall eines Auszubildenden aus Emden, der den mutmaßlichen Mörder eines Mädchens identifizierte und über Facebook zum Mord an dem vermeintlichen Mörder aufrief.

\footnotetext{
"Ab zur Polizeiwache, lasst uns das Schwein mit Steinen beschmeißen", schrieb er laut Staatsanwaltschaft wenige Tage nach Lenas Tod auf seiner Facebook-Seite. Anschließend versammelte sich ein Mob von rund 50 Menschen vor dem Emder Polizeikommissariat, in dem der Verdächtige saß. Sie riefen Sätze wie: "Schickt das Schwein raus. Dann werden wir uns um ihn kümmern."
}

(n. a. 2013) 
Der mutmaßliche Mörder erwies sich schließlich als unschuldig, der wahre Mörder wurde gefasst, der Denunziant zu Jugendarrest verurteilt. Weitere Fälle lassen sich leicht nachweisen.

Alle die genannten Formen legitimieren sich mit Bezug auf eine Institutionenkritik, also etwa "zu lasche Gesetze", "zu milde Strafverfolgung" oder eine Kombination daraus, sie schüren dabei eine hohe Emotionalität als Empörung, verbunden mit dem Bewusstsein, es selbst besser machen zu können, also besser beurteilen zu können, welche "Straftat" vorliege und welches "Urteil" über den "Angeklagten" zu sprechen sei - und dieses "Urteil" dann zur "Vollstreckung" an jeden auch tatsächlich freizugeben, womit der "Angeklagte" praktisch als vogelfrei erklärt wird. Es handelt sich also um Praktiken, die an die vorinstitutionellen Pogrome gegen Juden im Vorfeld und in der Frühphase des Nationalsozialismus erinnern (vgl. Wildt 2007). Selbstermächtigte Status-Degradierungszeremonien sind auch heute eine praktizierte Form von Rechtextremisten, wie dies im Verfassungsschutzbericht dokumentiert ist (vgl. Bundesministerium des Innern 2014: 63, 65) und in der Tagespresse verfolgt werden kann.

\section{$7 \quad$ Fazit}

Die Betrachtung der Mentalität der Selbstermächtigung gegenüber dem demokratisch legitimierten Rechtssystem im Bereich des Rechtswesens, einem konstitutiven Element der Demokratie, zeigt das große Gewaltpotenzial, das durch die Empörung über die "versagenden Institutionen", und zwar nicht nur die Judikative, sondern auch die Legislative und die Exekutive wachgerufen wird. Wie in anderen Bereichen geht mit der Empörung über die - so wahrgenommene - versagende (demokratische) Institution das (Selbst-)Bewusstsein der Eigenkompetenz einher, die als angemessener bzw. besser als die der Institutionenvertreter wahrgenommen und offensiv vertreten wird. Das Unbehagen, das sich bei der Interpretation der Daten zu selbstermächtigten Status-Degradierungszeremonien einstellt, liegt eben in dem Ausmaß an Aggression und Gewaltbereitschaft begründet, die durch die Hasssprache in den Kommentaren der "selbstermächtigten Justiz online" in großem Umfang erscheint. Es geht dabei nicht nur um das im Internet bekannte Flaming, das ja häufig bereits durch den Schutz der Anonymität hervorgerufen wird, sondern um die beschriebenen Eigenschaften der Mentalität der Selbstermächtigung, d. h. die Kombination der Empörung über die versagende Institution mit dem unbedingten Glauben an die eigene, den Institutionen überlegene Kompetenz, ihre angeblich "direkte" Verbindung zum "Volk", "Volkes Willen", "Gesetz" oder dem, was denn "Wissenschaft" ausmache, die sie in ihrer selbstermächtigten Repräsentation proklamieren.

Wenn Selbstermächtigung als Mentalität betrachtet wird, die sich zugleich mit der Demokratie konstituiert, ist diese nicht immer nur im Sinne emanzipatorischer Bewegungen zu denken, sondern sie liegt quer zu konkreten politischen Bewegungen und damit auch quer zu einem Links-Mitte-Rechts-Denken. Selbstermächtigung ist ambivalent, da sie nicht nur Selbstbestimmung beschreibt, sondern auch Selbstgerechtigkeit im Sinne der eben beschriebenen Selbstjustiz oder schlicht auch Ignoranz und Arroganz. 


\section{$8 \quad$ Desiderata}

In den vorliegenden Interpretationen der Fallstudien sind viele Fragen offen geblieben bzw. auch neu aufgeworfen worden, die in Zukunft bearbeitet werden müssten.

So müsste etwa das Verhältnis zwischen Skandal und Skandalisierung auf der einen und selbstermächtigten Status-Degradierungszeremonien auf der anderen Seite noch genauer untersucht werden: Zwar ist es offensichtlich, dass beide nicht identisch sind, doch wie das Verhältnis genau ist, ist noch ungeklärt. Eine selbstermächtigte Status-Degradierungszeremonie setzt wie der Skandal eine Normverletzung voraus, doch - darauf wurde schon zu Beginn hingewiesen - ist vieles, was in der Skandalforschung als Skandal betrachtet wird, keine selbstermächtigte Status-Degradierungszeremonie. Hier wäre insbesondere an die frühen Arbeiten Hitzlers (1987, 1989) anzuknüpfen, der Garfinkel als einer der wenigen Skandalforscher rezipiert hat.

Ebenfalls zu kurz kam die Entwicklung der Institutionen, insbesondere der demokratischen Institutionen: Böte die Institution nicht auch Angriffsflächen, indem sie die Funktion(en) nicht mehr erfüllt, die ihnen ihre Existenzberechtigung gibt, dann gäbe es auch nicht die Mentalität der Selbstermächtigung mit dem starken emotionalen, anti-institutionellen Impuls. Die Veränderungen staatlicher Institutionen, insbesondere auch demokratischer Institutionen sind eindringlich von Scharpf (1996) und Crouch (2008) beschrieben worden. Eine Betrachtung der Veränderung von Institutionen und ihrem Beitrag zur Mentalität der Selbstermächtigung muss hier allerdings als Desiderat stehen bleiben.

Schließlich kam auch die produktive Seite der Kulturen der Selbstermächtigung zu kurz, insbesondere, was die Formierung neuer Organisationsformen betrifft, die versuchen Stabilität und Fluidität in Balance zu halten (z. B. Creative Commons 2001-2015). Auch dies müsste unbedingt in einer umfangreicheren Betrachtung eingebracht werden.

\section{$9 \quad$ Ausblick: Die fünfte Gewalt}

Die Aufarbeitung der genannten Desiderata könnte einen Aspekt vertiefen, der in den Interpretationen mehrfach aufgefallen war, aber nicht vertieft werden konnte, nämlich das Zusammenspiel von traditionellen Medien und Neuen Medien im Rahmen selbstermächtigter Status-Degradierungszeremonien. Während der "Spill-Over" von Web-2.0-Medien in die traditionellen Meiden bekannt ist, ist jüngster Zeit das vorhin diskutierte Phänomen eines "SpillOver" von den traditionellen Medien in die Social Media beobachtet worden. So stellen Bieber, Härthe und Thimm (2015: 58) fest, dass diese beiden "Sphären immer engere Bezüge aufweisen":

Die gegenseitigen Bezugnahmen und Übernahmen von Themen verweisen darauf, dass der Spill-Over schneller und intensiver sein kann, als dies in früheren Zeiten der Social Meida der Fall war. Dies heißt jedoch, dass ein solches Modell zum Verständnis der Dynamiken solcher Social Media Kampagnen diese immer enger werdende Verzahnung der Medienspähren einbeziehen muss.

Dies verweist über die Phänomenkonstatierung hinaus auf eine mögliche kulturelle Verschiebung hin: Die Grenzen zwischen Gewaltmonopol und eigenmächtigem Handeln sind in der Geschichte immer wieder neu verhandelt worden (vgl. Foucault 1976/1994), zuletzt etwa in 
den 80er Jahren anlässlich der Blockaden des geplanten US-amerikanischen Pershing IIStandorts in Mutlangen oder auch in der Frage des so genannten "Kirchenasyls". Letztlich kann auch der investigative Journalismus, der mehr und mehr durch einen SkandalJournalismus ergänzt wurde, als eine Form bereits institutionalisierter Selbstermächtigung verstanden werden, daher die bildliche Redeweise von der "vierten Gewalt". Diese "vierte Gewalt" sieht sich nun - selbst in einer Krise institutioneller Erstarrung und veränderter Ressourcenverteilung - durch die Web-2.0-Medien von einer Vielzahl neuer sich selbst ermächtigender Akteure entmächtigt und erfährt die Notwendigkeit, sich neu zu positionieren.

Dies wird auch im traditionellen Journalismus reflektiert. So schreibt der Spiegelredakteur Cordt Schnibben (2015: 81) mit Blick auf eine Sichtung der Briefe, die er von seinen Lesern erhält:

Mehr oder minder pauschal werden Journalisten als willfährige Helfer dubioser Interessen abgekanzelt, im Chefarztton, das hat sich rumgedreht, und das Misstrauen in solchen Briefen und in unsere Arbeit - ist unübersehbar.

(Schnibben 2015: 81)

Ähnlich wie die Einführung von Massenprintverfahren und Demokratie zu einer Neuverteilung der Rechte und damit zum Entstehen des heutigen Journalismus führten, nämlich eines Journalismus, der unter dem Recht der Meinungs- und Zensurfreiheit öffentlich anklagen und - im Skandal-Journalismus - durchaus auch selbstermächtigte Status-Degradierungszeremonien durchführen darf - zumindest sofern es sich um Personen des öffentlichen Lebens handelt.

Mit der Einführung der sozialen Medien und der damit verbundenen "MassenAmateurisierung" (Shirky 2005: 15:12 min) könnte sich nun ein ähnlicher Kampf um eine Neu-Positionierung andeuten. Dieser Kampf verläuft nun aber nicht nur zwischen den Neuen Medien und dem Staat, sondern auch zwischen Neuen und traditionellen Medien. Wie also eine Neuordnung im Verhältnis von Rechtsstaat und selbstermächtigter Anklage in den Zeitungen und später im Fernsehen die Voraussetzung zur Entstehung der "vierten" Gewalt waren, sind die Einführung von Web 2.0-Technologien die Voraussetzung für die Entstehung einer fünften Gewalt. Während diese unterschiedlichen Aushandlungsprozesse derzeit in vollem Gang sind, erscheint die neue Ordnung, die sich daraus ergeben mag, derzeit nur ambivalent und unklar.

Auch die Institutionen haben erkannt, dass sich hier eine neue Macht konstituiert, die kaum mit traditionellen Begriffen zu fassen ist, weil die fluiden Formen der Netzwerke dem Paradigma der identitären Organisation nicht gehorcht. Sie reagieren in demokratischen Systemen mit einer Massenüberwachung aller Bürger, denn nur so können plötzliche Schwarm- und Netzwerkbildungen beobachtet werden. In autoritären Staaten werden Neue Medien dagegen schlichtweg zensiert oder verboten oder es werden eigene Propagandasender aufgebaut, die auch versuchen, das Netzwerk sozialer Medien zu beeinflussen. ${ }^{16}$

16 Nach der Druckfassung dieses Aufsatzes ist der Ausdruck "Fünfte Gewalt" prominent von Richard Gutjahr und Bernhard Pörksen verwendet worden, auf deren Ansätze hier aber leider nicht mehr eingegangen werden konnte. 
Wie bereits in den Desiderata angesprochen, sind die aggressiven und destruktiven Züge dieser entstehenden fünften Gewalt nur eine Seite, die andere Seite ist eine produktive und kommunitäre Seite, die in kreativer Weise auf die Spannungen der Spätmoderne reagiert. Eingangs wurde auf Sigmund Freuds "Unbehagen in der Kultur" (2004) verwiesen, die er mit den Worten beschloss:

Die Schicksalsfrage der Menschenart scheint mir zu sein, ob und in welchem Maße es ihrer Kulturentwicklung gelingen wird, der Störung des Zusammenlebens durch den menschlichen Aggressions- und Selbstvernichtungstrieb Herr zu werden.

(Freud 1930/2004: 108)

Selbst wenn man nicht wie Freud einen Aggressions- oder Todestrieb annimmt, sind die Neuen Medien zu Formen selbstermächtigter Aggressionsausübung geworden, die ein Unbehagen in der Demokratie entstehen lässt, und die Frage aufwirft, inwiefern Kultur noch Vergemeinschaftung in Frieden leisten kann. Dass die Erosion der Gemeinschaften und der Charaktere durch eine dialogisch gewonnene Verbindlichkeit der Commons-Kulturen aufgefangen werden kann, ist ebenso wie eine konstruktive Neuordnung der Gewalten eine Perspektive - doch bleibt Freuds Skepsis (ebd.): "Aber wer kann den Erfolg und Ausgang voraussehen?"

\section{Literatur}

Albrecht, Clemens et al. (2012): Was bedeutet Selbstermächtigung? www.autonomies.de [18.02.2015].

Bieber, Christoph/Härthe, Constantin/Thimm, Caja (2015): "Erregungskampagnen als transmediales Phänomen. Eine Medienanalyse von '\#aufschrei"'. In: Dies. (Hrsg): Erregungskampagnen in Politik und Wirtschaft. Digitale Öffentlichkeit zwischen Candy- und Shitstorms. Bonn, BAPP/IfAN: 47-58.

Bochinger, Christoph/Engelbrecht, Martin/Gebhardt, Winfried (2009): Die unsichtbare Religion in der sichtbaren Religion. Formen spiritueller Orientierung in der religiösen Gegenwartskultur. Stuttgart: Kohlhammer. (= Religionswissenschaft heute 3).

Butler, Judith (2013): Haß spricht. Zur Politik des Performativen. 4. Auflage. Berlin: Suhrkamp.

Crouch, Colin (2008): Postdemokratie. Frankfurt a. M.: Suhrkamp.

Deppermann, Arnulf (2005): Glaubwürdigkeit im Konflikt. Rhetorische Techniken in Streitgesprächen. Prozessanalysen von Schlichtungsgesprächen. Radolfzell, Verlag für Gesprächsforschung. www.verlag-gespraechsforschung.de/2005/pdf/konflikt.pdf [02.03.2015].

Duerr, Hans Peter (1993): Obszönität und Gewalt. Der Mythos vom Zivilisationsprozess. 3. Band. Frankfurt a. M.: Suhrkamp.

Felder, Ekkehard (2003): Juristische Textarbeit im Spiegel der Öffentlichkeit. Berlin/New York: de Gruyter. (= Studia Linguistica Germanica 70).

Foucault, Michel (1994): Überwachen und Strafen. Die Geburt des Gefängnisses. Frankfurt a. M.: Suhrkamp.

Freud, Sigmund (1930/2004): "Das Unbehagen in der Kultur." In: Ders.: Das Unbehagen in der Kultur und andere kulturtheoretische Schriften. 9. Auflage. Frankfurt a. M., Fischer: 29-108. (= Fischer Psychologie 10453).

Garfinkel, Harold (1956). "Conditions of Successful Degradation Ceremonies". American Journal of Sociology 61/5: 420-424. 
Gebhardt, Winfried (1993): "Idee, Mentalität, Institution. Kultursoziologische Anmerkungen zu einer Theorie institutionellen Wandels". Sociologia Internationalis. Internationale Zeitschrift für Soziologie, Kommunikations- und Kulturforschung 31/1993: 41-61.

Gebhardt, Winfried/Waldeyer, Christina (2011): "Das Bürgertum schlägt zurück. Le Dîner en Blanc als bürgerlicher Flashmob". In: Betz, Gregor/Hitzler, Ronald/Pfadenhauer, Michaela (Hrsg.): Urbane Events. Wiesbaden, VS: 227-244.

Grassmuck, Volker (2002): Freie Software: Zwischen Privat- und Gemeineigentum. Bonn: Bundeszentrale für politische Bildung.

Haller, André (2013): Dissens als kommunikatives Instrument: Theorie der intendierten Selbstskandalisierung in der politischen Kommunikation. Dissertation Universität Bamberg. http://opus4.kobv.de/opus4-bamberg/frontdoor/index/index/docId/3682 [21.02.2015].

Hermanns, Fritz (1995): "Sprachgeschichte als Mentalitätsgeschichte. Überlegungen zu Sinn und Form und Gegenstand historischer Semantik". In: Gardt, Andreas/Mattheier, Klaus J./Reichmann, Oskar (Hrsg.): Sprachgeschichte des Neuhochdeutschen. Gegenstände, Methoden, Theorien. Tübingen, Niemeyer: 69-101.

Hitzler, Ronald (1987): "Skandal: Karrierebremse oder Karrierevehikel? Inszenierungsprobleme Bonner Parlamentarier". Sozialwissenschaftliche Informationen 16/1: 22-27.

Hitzler, Ronald (1989): "Skandal ist Ansichtssache. Zur Inszenierungslogik ritueller Spektakel in der Politik". In: Ebbighausen, Rolf/Neckel, Sighard (Hrsg.): Anatomie des politischen Skandals. Frankfurt a. M., Suhrkamp: 334-354.

Kallmeyer, Werner/Schmitt, Reinhold (1996): "Forcieren oder: Die verschärfte Gangart. Zur Analyse von Kooperationsformen im Gespräch". In: Kallmeyer, Werner (Hrsg.): Gesprächsrhetorik. Tübingen, Narr: 9-118. (= Rhetorische Verfahren im Gesprächsprozeß 4).

Kämper, Heidrun (2013): Wörterbuch zum Demokratiediskurs 1967/68. Berlin: Akademie.

Kerner, Max (1996): Aufstand der Laien: Expertentum und Demokratie in der technisierten Welt. Aachen/Leipzig/Paris: Thouet.

Liebert, Wolf-Andreas (2015): "Metaphern der Selbstermächtigung. Max Stirners Philosophie des Einzigen als Bezugsstelle einer diskursiven Bewegung der Spätmoderne." In: Kämper, Heidrun/Warnke, Ingo (Hrsg.): Diskurslinguistik - Interdisziplinär. Zugänge, Gegenstände, Perspektiven. Berlin/Boston, de Gruyter: 121-144.

Lyotard, Jean-François (1999): Das postmoderne Wissen. Ein Bericht. 4. Auflage. Wien: Passagen. (=Edition Passagen 7).

Müller, Friedrich/Wimmer, Rainer (Hrsg.) (2001): Neue Studien zur Rechtslinguistik. Berlin: Duncker/Humblot. (= Schriften zur Rechtstheorie 202).

Pörksen, Bernhard/Detel, Hanne (2012): Der entfesselte Skandal. Das Ende der Kontrolle im digitalen Zeitalter. Köln: Halem.

Scharpf, Fritz W. (1996): "Demokratie in der transnationalen Politik". MPIfG Working Paper 96/3: 1-16. www.mpifg.de/pu/workpap/wp96-3/wp96-3.html [24.09.2014].

Sennett, Richard (1998): The Corrosion of Character. The Personal Consequences of Work in the New Capitalism. New York: Norton.

Shirky, Clay (2005): "Institutions vs. collaboration". TEDGlobal 2005. www.ted.com/talks/clay_shirky_on_institutions_versus_collaboration [06.03.2015]. 
Stötzel, Georg/Wengeler, Martin (1995): Kontroverse Begriffe. Geschichte des öffentlichen Sprachgebrauchs in der Bundesrepublik Deutschland. Berlin/New York: de Gruyter. (= Sprache, Politik, Öffentlichkeit 4).

Thomas, William Isaac/Thomas, Dorothy Swaine (1928): The Child in America: Behavior problems and programs. New York: Knopf. https://archive.org/details/ childinamerica00thom [26.02.2015].

Vogel, Friedemann (2012): Linguistik rechtlicher Normgenese. Theorie der Rechtsnormdiskursivität am Beispiel der Online-Durchsuchung. Berlin/Boston: de Gruyter. (= Sprache und Wissen 9)

Wengeler, Martin/Ziem, Alexander (Hrsg.) (2013): Sprachliche Konstruktionen von Krisen. Interdisziplinäre Perspektiven auf ein fortwährend aktuelles Phänomen. Bremen: Hempen. (= Sprache-Politik-Gesellschaft 12).

Wildt, Michael (2007): Volksgemeinschaft als Selbstermächtigung. Gewalt gegen Juden in der deutschen Provinz 1919 bis 1939. Hamburg: Hamburger Edition.

\section{Korpusverzeichnis}

Allianz der Wissenschaftsorganisationen (2014): Inakzeptable Grenzüberschreitung. Allianz der Wissenschaftsorganisationen wendet sich gegen diffamierende Zeitungsanzeigen gegen Neurowissenschaftler Professor Dr. Andreas Kreiter. www.dfg.de/download/pdf/dfg_im_ profil/reden_stellungnahmen/2014/stellungnahme_allianz_kreiter_140507.pdf [05.03.2015].

Becker, Markus (2014): "Empörung über Anzeigenkampagne gegen Hirnforscher". Spiegel Online. www.spiegel.de/wissenschaft/medizin/tierversuche-in-bremen-anzeigenkampagne-gegenkreiter-a-965656.html [06.03.2015].

Bidder, Benjamin (2014): "Internetaktivisten werfen ARD und ZDF antirussische Propaganda vor". Spiegel Online. http://www.spiegel.de/kultur/gesellschaft/ukraine-internet-aktivistenwerfen-ard-und-zdf-antirussische-propaganda-vor-a-994067.html [07.03.2015].

Bundesministerium des Innern (Hrsg.) (2014): Verfassungsschutzbericht 2013. www.verfassungsschutz.de/de/oeffentlichkeitsarbeit/publikationen/verfassungsschutzberic hte [06.03.2015].

Bundeszentrale für politische Bildung (Hrsg.) (2009): "Öffentlich-rechtlicher Rundfunk". Aus Politik und Zeitgeschichte 9-10/2009. www.bpb.de/apuz/32153/oeffentlich-rechtlicherrundfunk [26.02.2015].

Butscher (2014): "Einfach wütend". Forumsbeitrag zur Rede von Sibylle Lewitscharoff. www.klein-putz.net/forum/viewtopic.php?t=78951\&postdays=0\&postorder=asc\&\&pagi nation $=$ full\&start $=10$ [23.02.2015].

BVerwG (2014): Bundesverwaltungsgericht, Beschluss vom 20.01.2014 - 3 B 29.13. www.bverwg.de/200114B3B29.13.0 [05.03.2015].

Creative Commons (2001-2015): Creative Commons. https://creativecommons.org/ [06.03.2015].

Csuk, Cornelia (2014): "Aberkennung des Georg-Büchner-Kulturpreises für die Autorin Sybille Lewitscharoff". Openpetition. www.openpetition.de/petition/online/aberkennungdes-georg-buechner-kulturpreises-fuer-die-autorin-sybille-lewitscharoff-wegenunwuerdige [22.02.2015]. 
Csuk, Cornelia (2013a): CORNELIACSUK. My personal Blog. http://corneliacsuk. wordpress.com/ [22.02.2015].

Csuk, Cornelia (2013b): Leitfaden Reproduktionsmedizin: Wege zum Wunschkind. www.amazon.de/Leitfaden-Reproduktionsmedizin-Wege-zum-Wunschkind/dp/ 3732280616/ref=la_B00J69PB3M_1_1_bnp_1_pap?s=books\&ie=UTF8\&qid=142460924 $6 \& s r=1-1[22.02 .2015]$.

DiePresse.com (2014): Exkommunikation: Pfarrerinitiative "gegen Selbstermächtigung". http://diepresse.com/home/panorama/religion/3810995/Exkommunikation

_Pfarrerinitiative-gegen-Selbstermaechtigung [30.09.2014].

Flederjule (2014): "KiWu-Med. abartig? Kiwu-Kinder monströs? Protest erwünscht!!". Forumsbeitrag zur Rede von Sibylle Lewitscharoff. www.klein-putz.net/forum /viewtopic.php?t=78951 \\&sid=57c9f37ed53acb55af1c962f1de20dc7 [23.02.2015].

Gaertner, Rainer (2014): "Kreiter mach eiskalt weiter". Anzeige der Tierversuchsgegner Bundesrepublik Deutschland e. V. www.tierversuchsgegner.de/downloads/AZ-Kreiter-A3.pdf [05.03.2015].

Greiner, Lena/Demling, Alexander (2014): "Jugendliche gucken doch eh Pornos". Spiegel Online. www.spiegel.de/schulspiegel/sex-aufklaerung-forscherin-tuider-ueber-streit-umsexuellevielfalt-a-1001437.html [27.02.2015].

Katharinchen (2014): o.T. Forumsbeitrag zur Rede von Sibylle Lewitscharoff. www.kleinputz.net/forum/viewtopic.php?t=78951 \\&sid=57c9f37ed53acb55af1 c962f1 de20dc7 [23.02.2015].

Lewitscharoff, Sibylle (2014): Von der Machbarkeit. Die wissenschaftliche Bestimmung über Geburt und Tod. Dresdner Reden 2014, www.staatsschauspieldresden.de/download/18986/dresdner_rede_sibylle_lewitscharoff_final.pdf [30.09.2014].

Liebert, Wolf-Andreas (2014): \#Lewitscharoff-Tweets 06.03.-09.04.2014. Offline-Korpus, Universität Koblenz-Landau, Koblenz.

Mondschaf (2014): o.T. Forumsbeitrag zur Rede von Sibylle Lewitscharoff. www.kleinputz.net/forum/viewtopic.php?t=78951 \\&sid=57c9f37ed53acb55af1 c962f1 de20dc7 [23.02.2015].

Müller, Maren (2014): "Raus mit Markus Lanz aus meinem Rundfunkbeitrag!". OnlinePetition. www.openpetition.de/petition/online/raus-mit-markus-lanz-aus-meinerrundfunkgebuehr [24.02.2015].

n. a. (2013): "Mordfall Lena: 19-Jähriger wegen Aufruf zur Selbstjustiz verurteilt". Spiegel Online. www.spiegel.de/panorama/justiz/mordfall-lena-19-jaehriger-wegen-aufruf-zurselbstjustiz-verurteilt-a-905127.html [11.06.2013].

n. a. (2014a): Jeder Tag ohne dich, ist eine Qual für mich. www.facebook.com/AthirPageQual [05.03.2015]. Anmerkung: Mittlerweile trägt die Seite den Titel "Ohne dich geht die welt unter [sic]" [31.08.2015].

n. a. (2014b): Heute, den 07.03.2014 nominiere ich ihn als Offizieller Hurensohn des Jahres! Wer nominiert mit?! www.facebook.com/AthirPageQual/photos/pb.253311588184549.2207520000.1424994639./253317278183980/?type=3\&theater [05.03.2015].

Radio Blau (2014): Interview zur Markus Lanz-Petition mit Maren Müller am 21.1.2014 in Radio Blau. www.youtube.com/watch?v=dgJCB8Lbark [24.02.2015]. 
Radio Bremen (2014): Herr Kreiter sollte sich noch nicht in Hochstimmung befinden. Radio Bremen-Interview mit Wolfgang Apel. www.radiobremen.de/politik/themen/affenver suchebremen102.html [06.03.2015].

Schnibben, Cordt (2015): "Knast, wenn du lügst!". Der Spiegel 10: 81-86.

Scholz-Reiter, Bernd (2014): Offener Brief des Uni-Rektors an die Verlagsleitungen und Chefredaktionen von Die Zeit, FAZ, Tagesspiegel und Bremer Tageszeitungen AG. Universität Bremen www.uni-bremen.de/aktuelle-meldungen/einzelanzeige/news/detail/News /offener-brief-des-uni-rektors-an-die-verlagsleitungen-und-chefredaktionen-von-die-zeitfaz-tagessp.html?cHash=bd7ca92f26c3bade228b0f14dac22231 [05.03.2015].

Ständige Publikumskonferenz der öffentlich-rechtlichen Medien e. V. (2014): Ständige Publikumskonferenz der öffentlich-rechtlichen Medien. www.publikumskonferenz.de [24.02.2015].

Strick, Jasna Lisha (2013): "Ihr gehört nur mal ordentlich durchgevögelt". Hate Speech und Victim Blaming nach dem \#Aufschrei. Vortrag auf der openmind-Konferenz \#om13. Piratenpartei, Piraten Düren. http://youtu.be/wmR7cc4Pmt0 [06.03.2015].

SWR (2014): Internetvideo Hetzjagd auf Hundequäler. SWR-Landesschau Rheinland-Pfalz SWR. http://mp4-download.swr.de/swr-fernsehen/landesschau-rp/aktuell/2014/03/694830 .m.mp4 [11.03.2014].

Universität Kassel (2014): Stellungnahme zu den Äußerungen von Frau Sibylle Lewitscharoff. www.uni-kassel.de/uni/universitaet/pressekommunikation/publik-online/start/artikellesen/article/stellungnahme-zu-den-aeusserungen-von-frau-sibylle-lewitscharoff.html [30.09.2014]. 